Research Article

\title{
Applying Four-Step Characteristic Ion Filtering with HPLC-Q-Exactive MS/MS Spectrometer Approach for Rapid Compound Structures Characterization and Major Representative Components Quantification in Modified Tabusen-2 Decoction
}

\author{
Yu Zhao, Xin Dong, Zhi Wang, Rui Dong, Ren Bu, Qianxi Feng, \\ Peifeng Xue $\mathbb{D}^{\text {, }}$, and Bi Qu \\ Department of Pharmacy, Inner Mongolia Medical University, Jinshan Development Zone, Hohhot 010110, China
}

Correspondence should be addressed to Peifeng Xue; xpfdc153@163.com and Bi Qu; yxyqubi@163.com

Received 22 August 2021; Revised 19 November 2021; Accepted 26 November 2021; Published 31 December 2021

Academic Editor: Yingqiu Xie

Copyright $(2021$ Yu Zhao et al. This is an open access article distributed under the Creative Commons Attribution License, which permits unrestricted use, distribution, and reproduction in any medium, provided the original work is properly cited.

\begin{abstract}
Modified Tabusen-2 decoction (MTBD) is traditional Chinese Mongolia medicine, mainly used to treat osteoporosis. However, the precise material basis of this prescription is not yet fully elucidated. Herein, we establish an HPLC-Q-Exactive MS/MS spectrometer method with four-step characteristic ion filtering (FSCIF) strategy to quickly and effectively identify the structural features of MTBD and determine the representative compounds content. The FSCIF strategy included database establishment, characteristic ions summarization, neutral loss fragments screening, and secondary mass spectrum fragment matching four steps. By using this strategy, a total of 143 compounds were unambiguously or tentatively annotated, including 5 compounds which were first reported in MTBD. Nineteen representative components were simultaneously quantified with the HPLC-Q-Exactive MS/MS spectrometer, and it is suitable for eight batches of MTBD. Methodology analysis showed that the assay method had good repeatability, accuracy, and stability. The method established above was successfully applied to assess the quality of MTBD extracts. Collectively, our findings enhance our molecular understanding of the MTBD formulation and will allow us to control its quality in a better way. At the same time, this study can promote the development and utilization of ethnic medicine.
\end{abstract}

\section{Introduction}

Tabusen-2 decoction (TBD) is composed of Echinops latifolius Tausch (ELT) and Eucommia ulmoides Oliver (EU) [1]. On this basis, Modified Tabusen-2 decoction (MTBD) adds Panax notoginseng (PN) and Carthamus tinctorius L. (CT) [2]. Osteoporosis is a common orthopedic disease, especially in the elderly and postmenopausal women in China. TBD is a traditional classic prescription; it has been used to treat osteoporosis for centuries [3]. The literature shows that MTBD has the effect of treating osteoporosis; it can also be used to promote blood circulation, relieve swelling, relieve pain, continue muscles and bones, and treat soft tissue contusions, crush injuries, joint sprains, trauma, and open trauma caused by surgery $[4,5]$. The chemical compositions of each herb are various, having different pharmacological effects according to past reports. ELT, a traditional Chinese Mongolia herb, contained isochlorogenic acid A (ICGAA), chlorogenic acid, and other phenylpropanoids $[6,7]$, which are the main active components in herb. The pharmacological mitigation of ELT on osteoporosis of postmenopausal women was also reported [8]. EU is enriched with lignans and iridoids, including geniposidic acid (GPA) and pinoresinol diglucoside (PDG), having obvious antihypertensive effect [9]. In recent years, EU has attracted considerable attention because of its antiosteoporosis, antisenile dementia, antiaging, anti-inflammatory, antithrombotic, and antitumor activities $[10,11]$. The flavonoids are the main active components of $\mathrm{CT}$, with the efficacy of promoting blood circulation, 
removing blood stasis, and relieving pain [12]. Varieties of natural pigments isolated from $\mathrm{CT}$, such as yellow pigments and red pigments [13], not only have pharmacological functions but also have some nutritive value. Furthermore, triterpenoid saponins are main active constituents in PN, which are widely used for promoting blood clotting, relieving swelling, and alleviating pain [14].

In accordance with traditional Chinese medicine (TCM), traditional Mongolia medicine (TMM) is characterized with multiple components and multiple targets and plays different roles in clinical therapy. This means that it is a great challenge to explain the main chemical composition of MTBD by traditional analytical methods. In particular, the presence of isomers makes its separation and analysis more difficult. In order to solve this problem, some researchers have used the methods of mass defect, relative mass defect, neutral loss filtering (NLF), mass defect filtering, and precursor ion to characterize the chemical structure in TCM or TMM prescription [15-19]. It has vital-important reference value for our following experiment. With the promotion of high-resolution mass spectrometry $[20,21]$, we propose an FSCIF strategy for substructure recognition, which can significantly improve the detection effectiveness, accuracy, and sensibility. This analysis program shows obvious efficiency (reduce data processing time) and intelligence (simplify the process of structural identification).

Xie et al. [22] determined hydroxysafflor yellow A, notoginsenoside $R_{1}$, ginsenoside $\mathrm{Rg}_{1}$, and ginsenoside $R b_{1}$ with HPLC, but there are disadvantages of insufficient sensitivity and long running time (40 min). Hua et al. [23] established an HPLC-ELSD method to quantify the content of notoginsenoside $\mathrm{R}_{1}$, ginsenoside $\mathrm{Rg}_{1}$, and ginsenoside $\mathrm{Re}$ in $\mathrm{PN}$ but did not determine the content of the main components of ELT, EU, and CT. Hua et al. [24] conducted three different experiments by using HPLC, Ultraviolet detection, and ELSD methods and finally measured the content of representative components of ELT, EU, PN, and CT. But the shortcomings of this method are cumbersomeness and low responsiveness and they cannot be ignored. On the other hand, the previous literature has qualitatively analyzed the ingredients in a single medicinal material; it is not enough to explain the overall structure of MTBD due to the interaction between temperature and herbs in the process of decoction.

In order to explore the material basis of MTBD, clarify the composition of the compounds, and determine the content of the compounds, this experiment used the HPLCQ-Exactive MS/MS spectrometer method to conduct a comprehensive material basis determination of MTBD, which provided a foundation for the subsequent quality standard formulation; it also provided guarantee for pharmacodynamic and pharmacokinetic research. Besides, 143 compounds were unambiguously or tentatively annotated with FSCIF strategy, including 5 compounds which were first reported in MTBD. Finally, we evaluated the differences in the content of 19 compounds in samples from different preparation batches, laying a foundation for subsequent quality evaluation.

\section{Experimental}

2.1. Materials and Reagents. A total of four batches of ELT were collected from various areas of Inner Mongolia (including Hohhot, Ordos, Xilingol, and Ulan Hot) in August 2020 (the GPS coordinates of the plant Echinops latifolius Tausch collection site are 41.1206962700 and 111.4084477500). Different batches of EU, CT, and PN herbs were purchased from Bozhou Pharmaceutical Co., Ltd. (Anhui, China) and GuoDa Drugstore (Hohhot, Inner Mongolia). All herbs were authenticated by Professor Bi Qu (Department of Pharmacognosy, Inner Mongolia Medical University). These specimens were preserved in the Department of General Investigation of Traditional Chinese Medicine Resources, Inner Mongolia Medical University.

Isochlorogenic acid A (ICGAA), 1,5-dicaffeoylquinic acid (1,5-DQA), genistein (GE), apigenin (APG), luteolin (LT), kaempferol (KPF), quercetin (QC), apigenin-7-Oglucuronide (A-7-0-G), rutin (RU), hydroxysafflor yellow A (HSYA), notoginsenoside $\mathrm{R}_{1}\left(\mathrm{NG}-\mathrm{R}_{1}\right)$, ginsenoside $\mathrm{Re}(\mathrm{G}-$ $\mathrm{Re})$, ginsenoside $\mathrm{Rg}_{1}\left(\mathrm{G}-\mathrm{Rg}_{1}\right)$, ginsenoside $\mathrm{Rb}_{1}\left(\mathrm{G}-\mathrm{R} \mathrm{b}_{1}\right)$, caffeic acid (CA), ferulic acid (FA), geniposidic acid (GPA), chlorogenic acid (CGA), pinoresinol diglucoside (PDG), and digoxin (internal standard, IS) were purchased from Cybertech Limited (Beijing, China), with HPLC purity $\geq 98 \%$. The chemical structures of these 19 compounds are displayed in Figure 1. LC-MS grade methanol, acetonitrile, and formic acid were achieved from Fisher Scientific (Hampton, NH, USA). Deionized water was prepared on a Millipore water purification system (Billerica, MA, USA). The columns used in the experiment were as follows: ACE C18-PFP column $(100 \times 3.0 \mathrm{~mm}$ ID, $3 \mu \mathrm{m})$, Grace Alltima C18 column $(250 \mathrm{~mm} \times 4.6 \mathrm{~nm}, 5 \mu \mathrm{m})$, HITACHI LaChrom C18 column $(250 \mathrm{~mm} \times 4.6 \mathrm{~mm} \mathrm{ID,} 5 \mu \mathrm{m})$, and Thermo ODS-2 HYPERSIL column $(250 \mathrm{~mm} \times 4.6 \mathrm{~mm}, 5 \mu \mathrm{m})$.

\subsection{MTBD Sample and Standard Solutions Preparation.} Sample preparation was a critical step for precise and convincing detection by the HPLC-Q-Exactive MS/MS spectrometer method. The MTBD samples were prepared according to our previous extraction process, and the whole operation process was in line with the basic operation safety regulations of the laboratory. EU, ELT, and CT herbal materials were powdered and sieved through 40 meshes for later extraction. A total $3.6 \mathrm{~g}$ of MTBD powders was accurately weighed (including $1.6 \mathrm{~g}$ of EU, $1.2 \mathrm{~g}$ of ELT, and $0.8 \mathrm{~g}$ of $\mathrm{CT}$ ) and placed in a $250 \mathrm{~mL}$ round-bottomed flask. These powders were immersed in $50 \mathrm{~mL}$ ethanol: water $(6: 4$, $\mathrm{V} / \mathrm{V})$ mixture and weighed and then reflux extracted twice, 


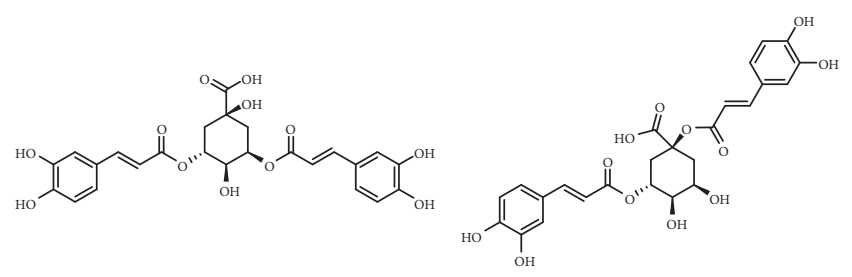

ICGAA<smiles>Cc1ccc(-c2ccc(I)cc2)c(C)c1</smiles><smiles>Cc1ccc(C2(C)CC3CCC(C)CC32C)cc1</smiles><smiles>Cc1ccc(C(C)C)c(C(C)C)c1</smiles>

APG<smiles>CC1O[C@H](OC[C@H]2O[C@H](Oc3oc4c(O)cc(O)cc4c(=O)c3-c3ccc(O)c(O)c3)[C@@H](O)[C@H](O)[C@H]2O)[C@H](O)[C@@H](O)[C@H]1O</smiles>

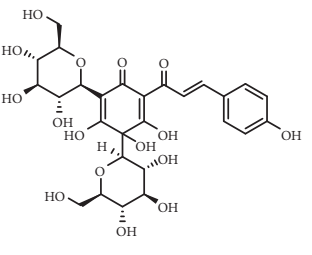

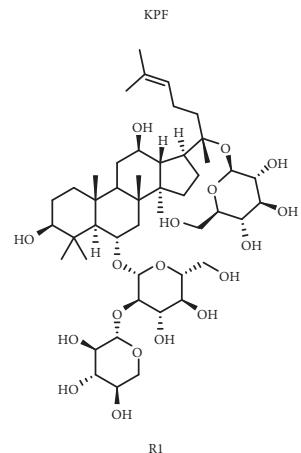<smiles>CC(C)CCC1CC(C)C(C)C1</smiles>

QC

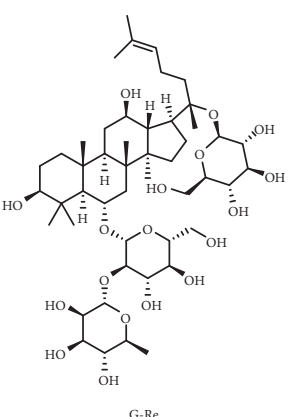<smiles>CCc1cc(CC=C(C)C)ccc1OC</smiles>
CA

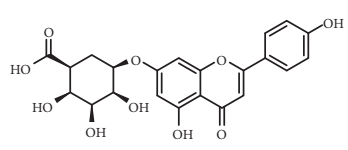

A-7-O-G

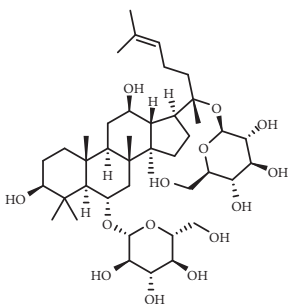

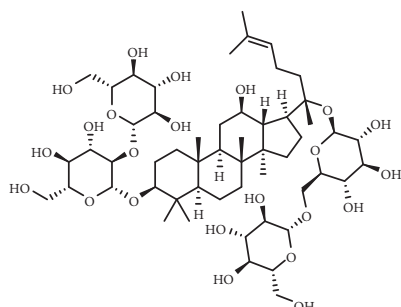<smiles>O=C(O)C1=CO[C@H](O[C@H]2OC(CO)[C@@H](O)[C@H](O)[C@H]2O)C2CC=C(CO)[C@H]12</smiles>

GPA<smiles>O=C(/C=C/c1ccc(O)c(O)c1)O[C@H]1C[C@H](C(=O)O)C[C@@H](O)[C@H]1O</smiles>

CGA

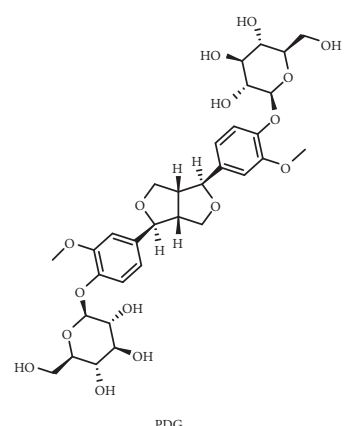

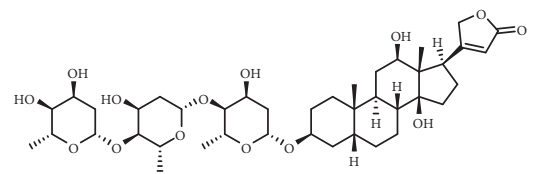

FIGURE 1: The chemical structures of nineteen analytes: isochlorogenic acid A (ICGAA), 1,5-dicaffeoylquinic acid (1,5-DQA), genistein (GE), apigenin (APG), luteolin (LT), kaempferol (KPF), quercetin (QC), apigenin-7-O-glucuronide (A-7-0-G), rutin (RU), hydroxysafflor yellow A (HSYA), notoginsenoside $R_{1}\left(N G-R_{1}\right)$, ginsenoside $R e(G-R e)$, ginsenoside $\operatorname{Rg}_{1}\left(G-R_{1}\right)$, ginsenoside $R b_{1}\left(G-R b_{1}\right)$, caffeic acid (CA), ferulic acid (FA), geniposidic acid (GPA), chlorogenic acid (CGA), pinoresinol diglucoside (PDG), and digoxin (internal standard, IS).

$90 \mathrm{~min}$ for each reflux. Taking into account the recovery rate of PN powder, $0.4 \mathrm{~g}$ PN was added before the last extraction. After merging and mixing, the solution was filtered through a $0.45 \mu \mathrm{m}$ microporous membrane. This filtrate was diluted 40 times for HPLC-Q-Exactive MS/MS spectrometer injection.

ICGAA $20.05 \mathrm{mg}, 1,5$-DQA $11.92 \mathrm{mg}$, GE $4.03 \mathrm{mg}$, APG $2.15 \mathrm{mg}$, LT $4.03 \mathrm{mg}$, KPF $1.30 \mathrm{mg}$, QC $2.02 \mathrm{mg}$, A-7-
O-G $3.85 \mathrm{mg}, \quad \mathrm{RU} \quad 4.23 \mathrm{mg}$, HSYA $19.80 \mathrm{mg}, \mathrm{NG}-\mathrm{R}_{1}$ $10.02 \mathrm{mg}, \quad \mathrm{G}-\mathrm{Re} \quad 19.40 \mathrm{mg}, \quad \mathrm{G}-\mathrm{Rg}_{1} \quad 10.17 \mathrm{mg}, \quad \mathrm{G}-\mathrm{Rb}_{1}$ $20.49 \mathrm{mg}$, CA $2.07 \mathrm{mg}$, FA $1.05 \mathrm{mg}$, GPA $4.05 \mathrm{mg}$, CGA $18.90 \mathrm{mg}$, and PDG $23.40 \mathrm{mg}$ were accurately weighted and transferred into $2 \mathrm{~mL}$ volumetric flask, respectively. Owing to the solubility of these compounds, methanol was applied to prepare the standard solution. In order to improve the precision and accuracy of the content, 
digoxin was selected as the internal standard. These standard solutions were diluted with mobile phase to final concentration (Table S1) before injection into HPLC-QExactive MS/MS spectrometer.

\subsection{Chromatography and Mass Spectrometry Conditions.} The characterization and quantification of MTBD sample extracts were analyzed using a Thermo HPLC-Q-Exactive MS/MS spectrometer system (HPLC, UltiMate 3000, mass system, Quadrupole Exactive Orbitrap ${ }^{\mathrm{TM}}$ ). The qualitative analytical conditions were as follows: HPLC column, COSMOSIL C18 $(250 \mathrm{~mm} \times 4.6 \mathrm{~mm}$ ID, $5 \mu \mathrm{m})$; solvent system, methanol $(\mathrm{A})$, and water containing $0.1 \%(\mathrm{v} / \mathrm{v})$ formic acid (B); gradient program, $0-5 \mathrm{~min}, 2 \%-5 \% \mathrm{~A}$; 5-10 min, 5\%-10\%A; $10-15 \mathrm{~min}, 10 \%-18 \% \mathrm{~A} ; 15-25 \mathrm{~min}$, 18\%-23\%A; 25-35 min, 23\%-28\%A; 35-55 min, 28\%-33\%A; 55-60 min, 33\%-39\%A; 60-70 min, 39\%-43\%A; 70-75 min, 43\%-46\%A; 75-85 min, 46\%-60\%A; 85-100 min, 60\%-65\%A; 100-105 min, $\quad 65 \%-75 \% \mathrm{~A} ; \quad 105-110 \mathrm{~min}, \quad 75 \%-100 \% \mathrm{~A}$; 110-130 $\mathrm{min}, 100 \%-100 \% \mathrm{~A}$; flow rate, $0.6 \mathrm{~mL} / \mathrm{min}$; column temperature, $30^{\circ} \mathrm{C}$; sample injection volume, $10 \mu \mathrm{L}$. The quantitative analysis of MTBD sample extracts was separated on an ACE C18-PFP $(100 \times 3.0 \mathrm{~mm}$ ID, $3 \mu \mathrm{m})$ column. The mobile phase consisted of methanol (A) and water containing $0.3 \%(\mathrm{v} / \mathrm{v})$ formic acid (B). A gradient program was used as follows: $0-6 \mathrm{~min}, 40 \%-40 \% \mathrm{~A}$; 6-15 min, 40\%-90\%A; 15-16 min, 90\%-10\%A; 16-21 min, $10 \%-10 \% \mathrm{~A} ; 21-22 \mathrm{~min}, 10 \%-40 \% \mathrm{~A}$; and $22-25 \mathrm{~min}, 40 \%-$ $40 \% \mathrm{~A}$. The flow rate was set as $0.3 \mathrm{~mL} / \mathrm{min}$. The column temperature was kept at $30^{\circ} \mathrm{C}$. Sample injection volume was $2 \mu \mathrm{L}$.

The qualitative and quantitative mass parameters conditions were set up as follows: auxiliary gas heater temperature, $150^{\circ} \mathrm{C}$; capillary temperature, $350^{\circ} \mathrm{C}$; spray voltage, $3.5 \mathrm{kv}$; S-lens RF level, 50; sheath gas flow rate, $40 \mathrm{~L}$; and auxiliary gas flow rate, 2 PSI. AGC was $3 \times 10^{6}$ in MS scan and $1 \times 10^{5}$ in MS/MS scan; IT was $100 \mathrm{~ms}$ in MS scan and $50 \mathrm{~ms}$ in MS/MS scan; resolution was 70000 in MS scan and 17500 in MS/MS scan; NCE was set as $30 \mathrm{v}$. Scanning range was $100-1500 \mathrm{~m} / z$. Mass spectrometry uses full scan mode for analysis in positive ion mode and negative ion mode.

2.4. Method Validation. The dependent variable was the ratio of the peak area of each analyte to the peak area of the internal standard, while the independent variable was set as the concentration value of each analyte; the least square regression was used to construct the standard curve equation. The intraday and interday precisions and accuracies were assessed by analyzing each concentration level (low, medium, and high) of six repeated QC samples on the same day and three consecutive days, respectively. Sample stability was investigated after the extracts were kept at room temperature for $0 \mathrm{~h}, 6 \mathrm{~h}, 12 \mathrm{~h}$, and $24 \mathrm{~h}$. Add the mixed control solution equal to the content of each analyte in the sample to the MTBD sample, repeat the preparation of 6 solutions, and calculate the recovery according to the following formula: recovery $(\%)=\frac{(\text { detected amount }- \text { original amount })}{\text { spiked amount }} \times 100 \%$

\section{Results and Discussion}

3.1. Construction of the Identification Strategy. Each type of compounds has its similar core and skeleton. On this basis, the characteristic ion will be produced, which provides us with new ideas for identifying these structures. In addition, FSCIF is especially suitable for compounds with the same structural type containing similar fragmentation pathways with some characteristic ions. Correspondingly, an FSCIFbased and substructure scanning strategy will be used for rapid identification of MTBD structures. The analytical strategy is shown in Figure 2. The compounds in MTBD were characterized by HPLC-Q-Exactive MS/MS spectrometer method with FSCIF strategy, including the following steps: (1) established the self-building chemical database of MTBD according to literature and online database; (2) comprehensively summarized characteristic ions for each compound type to conduct global identification of the ingredients in MTBD; (3) rapidly screened relevant structure information by neutral loss fragments (NLF) to conform the sugar type, conjunction position, and other information; (4) concluded the precise compound structure through high-precision MS/MS data. The typical total ion chromatograms (TICs) of MTBD by HPLC-Q-Exactive MS/MS spectrometer system in positive and negative ion modes are shown in Figure 3. 143 compounds were annotated through high-precision MS/MS data, including 51 triterpenoid saponins, 28 flavonoids, 20 phenylpropanoids, 15 iridoids, 12 lignans, 11 polyphenols, and 6 other types (Table 1), in which 5 compounds were first reported in MTBD and 20 compounds were unambiguously identified by comparison with reference standards. These $143 \mathrm{com}$ ponents' structures are shown in Figure S1.

\subsection{Qualitative Analysis}

3.2.1. Identification of Triterpenoid Saponins. Triterpenoid saponins were typical bioactive components of $\mathrm{PN}$, which were classified into two categories of protopanaxadiol (PPD) triterpenoid saponins and protopanaxatriol (PPT) triterpenoid saponins; the characteristic

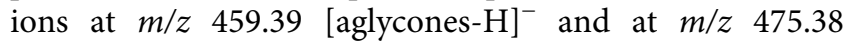
[aglycones- $\mathrm{H}]^{-}$corresponded to the PPD and PPT type ginsenosides [25]. In this study, most triterpenoid saponins (46 compounds) were detected $[\mathrm{M}+\mathrm{Na}]^{+}$in positive ion mode, other triterpenoid saponins (5 compounds) were detected $[\mathrm{M}-\mathrm{H}]^{-}$in negative ion mode, and excimer ion peaks can produce different cleavage modes to provide structural information such as aglycone type, sugar type, and its junction position. Compounds 110 and 123 were filtered by characteristic ion $m / z 459.39$, which tentatively identified PPD type ginsenosides; for compound $110\left(\mathrm{C}_{54} \mathrm{H}_{92} \mathrm{O}_{23}\right)$ $[\mathrm{M}-\mathrm{H}]^{-}$at $m / z 1107.5956$, its molecular ion peak successively lost the four molecules of glucose and obtained $\mathrm{m} / \mathrm{z}$ 


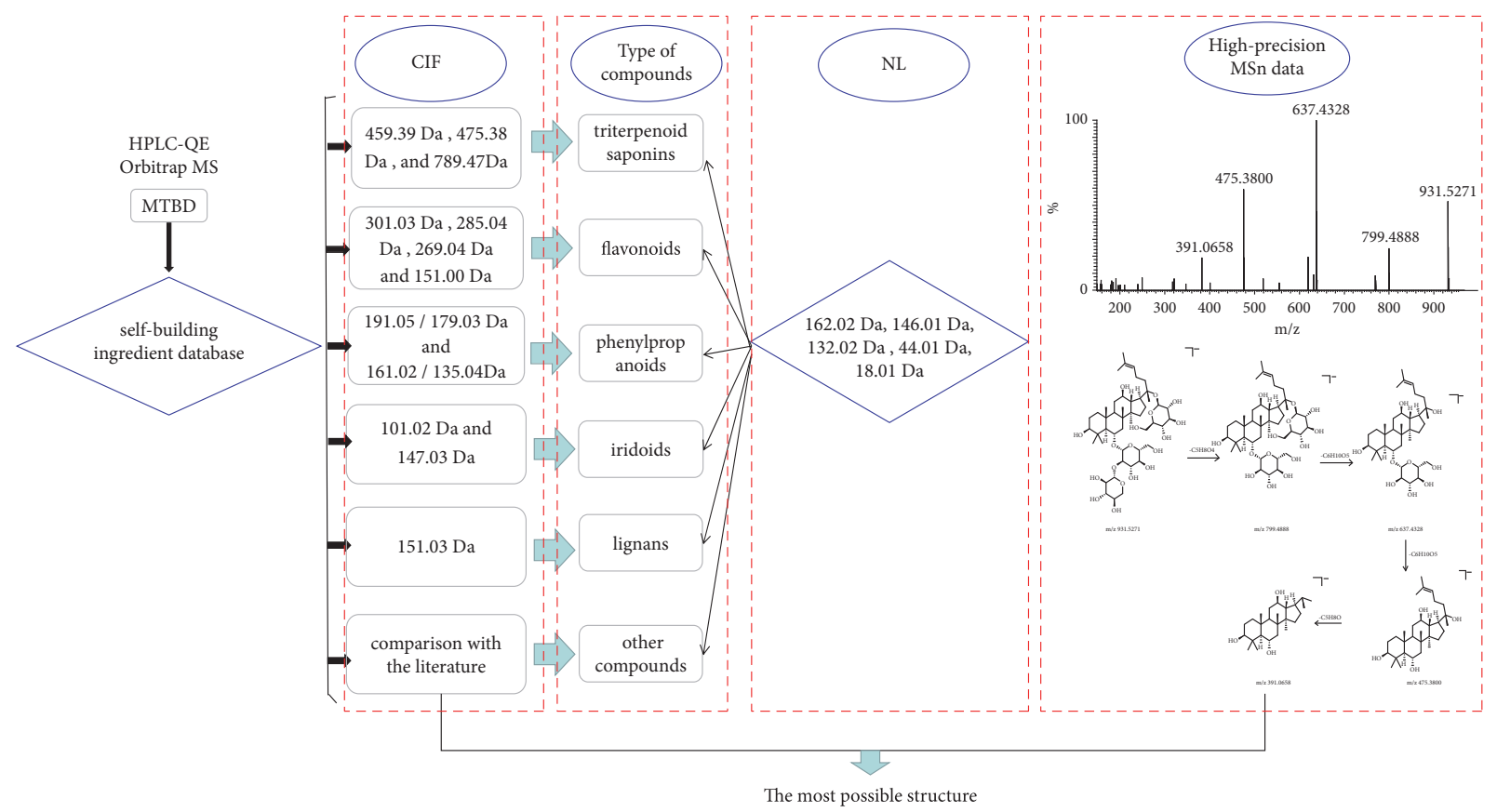

FIgUre 2: Analysis strategy of qualitative research of MTBD.

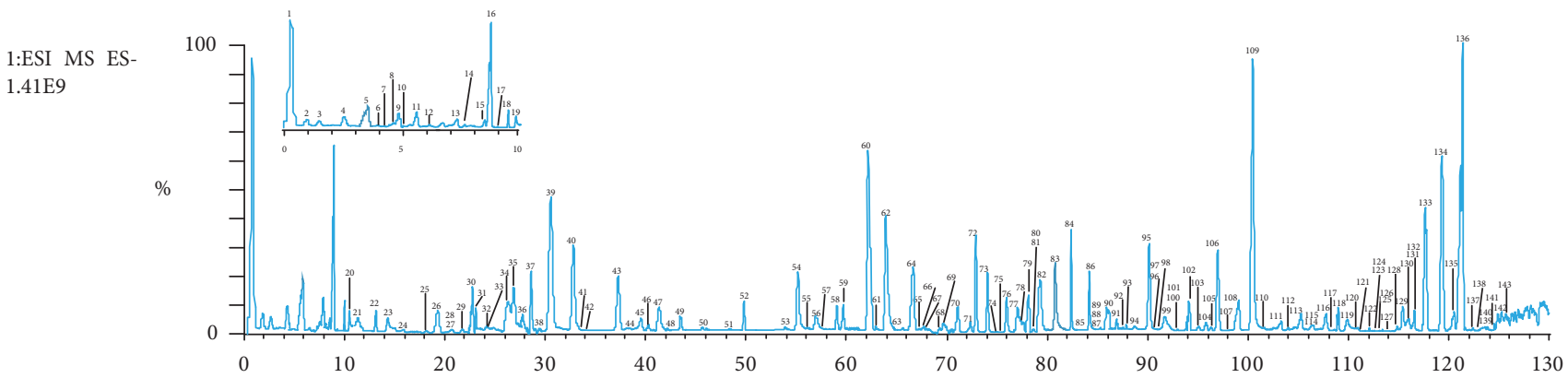

(a)

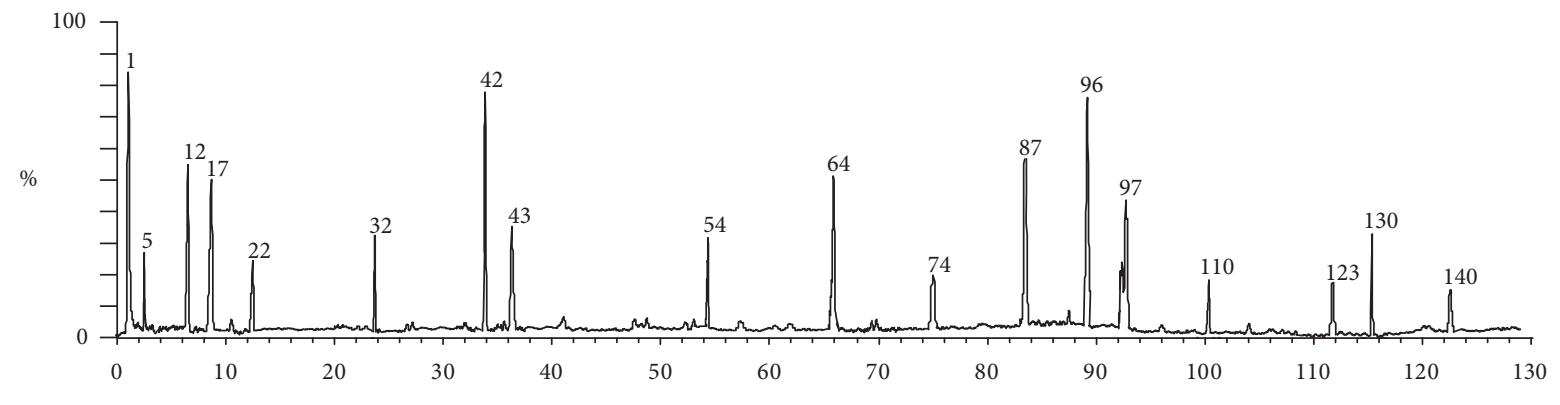

(b)

Figure 3: Continued. 


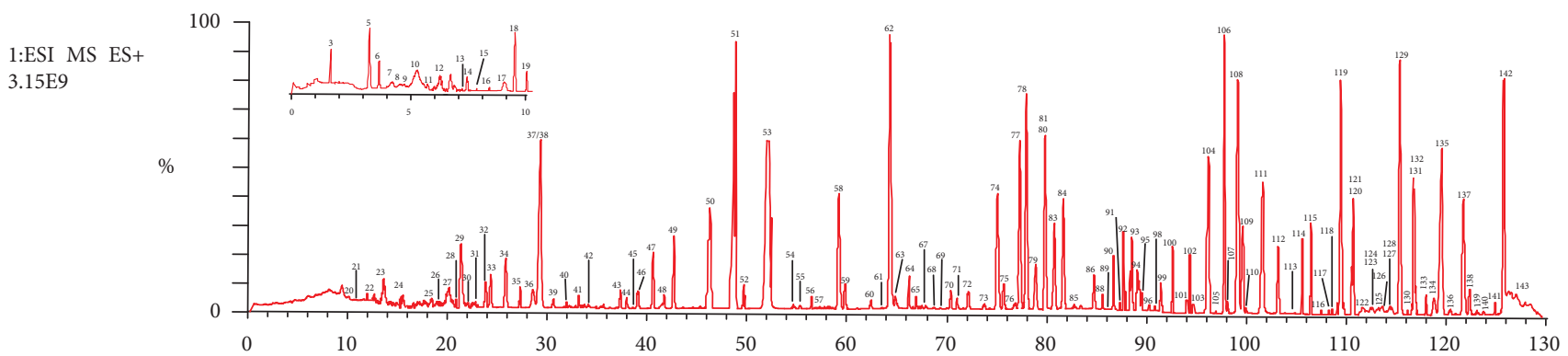

(c)

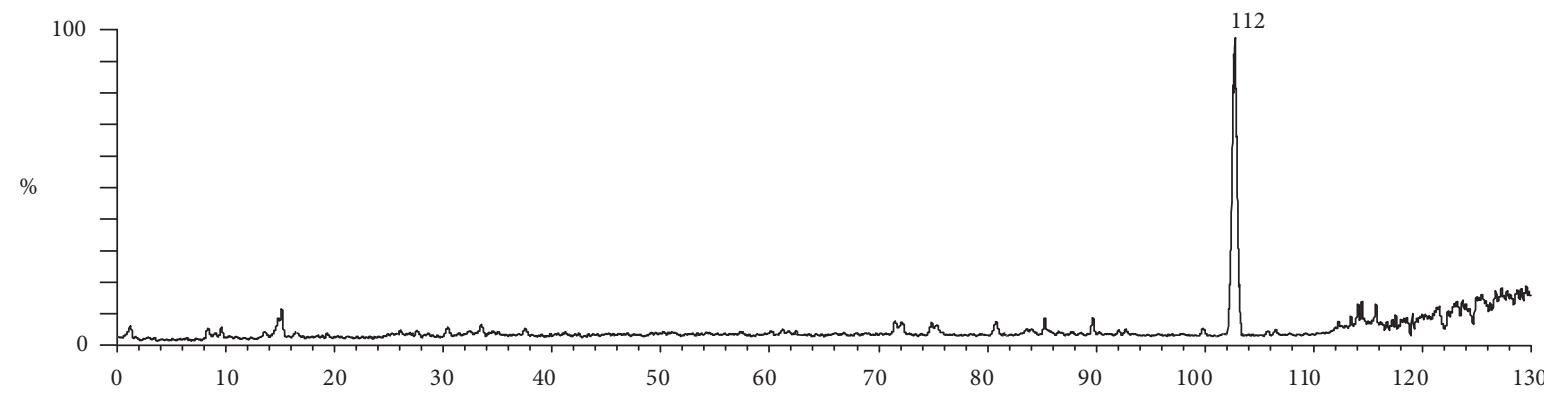

(d)

Figure 3: The typical total ion chromatograms (TICs) of MTBD. (a) TIC in negative ion mode. (b) Comparison with standard in negative ion mode. (c) TIC in positive ion mode. (d) Comparison with standard in positive ion mode.

TABLE 1: Characterization of chemical constituents of MTBD.

\begin{tabular}{|c|c|c|c|c|c|c|c|c|}
\hline No. & $\begin{array}{c}\mathrm{tR} \\
(\mathrm{min})\end{array}$ & Formula & Identification & $\begin{array}{l}\text { Precursor } \\
\text { ions }(m / z)\end{array}$ & $\begin{array}{c}\text { Diff } \\
(\mathrm{ppm})\end{array}$ & $\begin{array}{l}\text { Fragment } \\
\qquad(\mathrm{m} / \mathrm{z})\end{array}$ & Type & $\begin{array}{l}\text { Reference } \\
\text { standard }\end{array}$ \\
\hline 1 & 0.25 & $\mathrm{C}_{27} \mathrm{H}_{30} \mathrm{O}_{16}$ & Rutin & $\begin{array}{l}609.1461 \\
{[\mathrm{M}-\mathrm{H}]^{-}}\end{array}$ & -3.414 & $\begin{array}{c}301.0351 \\
300.0278, \\
283.0325, \\
271.0251 \\
255.0292 \\
227.0321 \\
151.0293\end{array}$ & $\mathrm{~F}$ & Yes \\
\hline 2 & 1.05 & $\mathrm{C}_{15} \mathrm{H}_{10} \mathrm{O}_{7}$ & Quercetin & $\begin{array}{l}301.0353 \\
{[\mathrm{M}-\mathrm{H}]^{-}}\end{array}$ & 3.724 & $\begin{array}{l}273.0405 \\
257.0452 \\
229.0500, \\
178.9978, \\
151.0026 \\
121.0283 \\
107.0126\end{array}$ & $\mathrm{~F}$ & \\
\hline 3 & 1.68 & $\mathrm{C}_{8} \mathrm{H}_{8} \mathrm{O}_{4}$ & Vanillic acid & $\begin{array}{l}167.0349 \\
{[\mathrm{M}-\mathrm{H}]^{-}}\end{array}$ & 0.867 & $\begin{array}{c}152.0105 \\
123.0438 \\
108.0203\end{array}$ & $\mathrm{PO}$ & \\
\hline 4 & 2.35 & $\mathrm{C}_{17} \mathrm{H}_{20} \mathrm{O}_{9}$ & Methyl chlorogenic acid & $\begin{array}{l}367.1034 \\
{[\mathrm{M}-\mathrm{H}]^{-}}\end{array}$ & 5.239 & $\begin{array}{l}191.0553 \\
173.0078\end{array}$ & $\mathrm{P}$ & \\
\hline 5 & 3.57 & $\mathrm{C}_{11} \mathrm{H}_{14} \mathrm{O}_{5}$ & Genipin & $\begin{array}{l}225.0768 \\
{[\mathrm{M}-\mathrm{H}]^{-}}\end{array}$ & 2.738 & $\begin{array}{c}207.0659 \\
147.0441 \\
123.0439 \\
101.0231\end{array}$ & I & Yes \\
\hline 6 & 3.82 & $\mathrm{C}_{6} \mathrm{H}_{6} \mathrm{O}_{3}$ & Pyrogallic acid & $\begin{array}{l}125.0244 \\
{[\mathrm{M}-\mathrm{H}]^{-}}\end{array}$ & -1.604 & $\begin{array}{c}107.4741 \\
97.0282\end{array}$ & $\mathrm{PO}$ & \\
\hline 7 & 4.09 & $\mathrm{C}_{16} \mathrm{H}_{22} \mathrm{O}_{10}$ & Geniposidic acid & $\begin{array}{l}373.1140 \\
{[\mathrm{M}-\mathrm{H}]^{-}}\end{array}$ & -6.227 & $\begin{array}{c}211.0940 \\
193.0498, \\
167.0703, \\
149.0598 \\
123.0439\end{array}$ & I & \\
\hline
\end{tabular}


TABle 1: Continued.

\begin{tabular}{|c|c|c|c|c|c|c|c|c|}
\hline No. & $\begin{array}{c}\mathrm{tR} \\
(\mathrm{min})\end{array}$ & Formula & Identification & $\begin{array}{l}\text { Precursor } \\
\text { ions }(\mathrm{m} / \mathrm{z})\end{array}$ & $\begin{array}{c}\text { Diff } \\
(\mathrm{ppm})\end{array}$ & $\begin{array}{c}\text { Fragment } \\
(\mathrm{m} / \mathrm{z})\end{array}$ & Type & $\begin{array}{c}\text { Reference } \\
\text { standard }\end{array}$ \\
\hline 8 & 4.47 & $\mathrm{C}_{16} \mathrm{H}_{22} \mathrm{O}_{11}$ & Deacetyl asperulosidic acid & $\begin{array}{l}389.1089 \\
{[\mathrm{M}-\mathrm{H}]^{-}}\end{array}$ & 3.167 & $\begin{array}{l}227.0550 \\
209.0356 \\
191.0553 \\
183.0655 \\
165.0541 \\
147.0285 \\
139.0389\end{array}$ & I & \\
\hline 9 & 4.85 & $\mathrm{C}_{10} \mathrm{H}_{14} \mathrm{O}_{10}$ & 2-Methylsuccinyl-6'-O-glucoside & $\begin{array}{l}293.0514 \\
{[\mathrm{M}-\mathrm{H}]^{-}}\end{array}$ & 6.047 & 131.0450 & $\mathrm{PO}$ & \\
\hline 10 & 4.92 & $\mathrm{C}_{15} \mathrm{H}_{22} \mathrm{O}_{9}$ & Aucubin & $\begin{array}{l}345.1191 \\
{[\mathrm{M}-\mathrm{H}]^{-}}\end{array}$ & 2.335 & $\begin{array}{c}183.0660 \\
165.0543 \\
139.0391 \\
121.0285\end{array}$ & I & \\
\hline 11 & 5.37 & $\mathrm{C}_{17} \mathrm{H}_{26} \mathrm{O}_{11}$ & Harpagide acetate & $\begin{array}{l}405.1402 \\
{[\mathrm{M}-\mathrm{H}]^{-}}\end{array}$ & -1.364 & $\begin{array}{c}191.0554 \\
147.0289 \\
119.0026 \\
101.0023\end{array}$ & I & \\
\hline 12 & 6.23 & $\mathrm{C}_{15} \mathrm{H}_{10} \mathrm{O}_{6}$ & Kaempferol & $\begin{array}{l}285.0404 \\
{[\mathrm{M}-\mathrm{H}]^{-}}\end{array}$ & 4.615 & $\begin{array}{l}257.0453 \\
243.1601 \\
239.1650 \\
229.0322 \\
199.0395 \\
185.0420\end{array}$ & $\mathrm{~F}$ & Yes \\
\hline 13 & 7.41 & $\mathrm{C}_{23} \mathrm{H}_{34} \mathrm{O}_{15}$ & Genipin gentian diglycoside & $\begin{array}{l}549.1824 \\
{[\mathrm{M}-\mathrm{H}]^{-}}\end{array}$ & 5.378 & $\begin{array}{l}387.2035 \\
207.1128 \\
179.0551 \\
147.0298\end{array}$ & I & \\
\hline 14 & 7.50 & $\mathrm{C}_{25} \mathrm{H}_{24} \mathrm{O}_{11}$ & 3-Caffeoyl-5-coumaroyl-quinic acid & $\begin{array}{l}499.12458 \\
{[\mathrm{M}-\mathrm{H}]^{-}}\end{array}$ & 5.81 & $\begin{array}{l}353.1080 \\
191.0554\end{array}$ & $\mathrm{P}$ & \\
\hline 15 & 8.32 & $\mathrm{C}_{19} \mathrm{H}_{18} \mathrm{O}_{11}$ & Isomangiferin & $\begin{array}{l}421.0776 \\
{[\mathrm{M}-\mathrm{H}]^{-}}\end{array}$ & -1.966 & 259.0224 & $\mathrm{~F}$ & \\
\hline 16 & 8.44 & $\mathrm{C}_{9} \mathrm{H}_{6} \mathrm{O}_{3}$ & Umbelliferone & $\begin{array}{l}161.0244 \\
{[\mathrm{M}-\mathrm{H}]^{-}}\end{array}$ & -0.249 & $\begin{array}{c}135.0441 \\
99.0438 \\
71.0124\end{array}$ & $\mathrm{P}$ & \\
\hline 17 & 8.99 & $\mathrm{C}_{7} \mathrm{H}_{6} \mathrm{O}_{5}$ & Gallic acid & $\begin{array}{l}169.0142 \\
{[\mathrm{M}-\mathrm{H}]^{-}}\end{array}$ & 0.179 & $\begin{array}{c}125.0232 \\
141.0914\end{array}$ & $\mathrm{PO}$ & Yes \\
\hline 18 & 9.25 & $\mathrm{C}_{6} \mathrm{H}_{6} \mathrm{O}_{4}$ & 2-Hydroxyphenol & $\begin{array}{l}141.0193 \\
{[\mathrm{M}-\mathrm{H}]^{-}}\end{array}$ & 3.247 & 123.0175 & $\mathrm{PO}$ & \\
\hline 19 & 9.76 & $\mathrm{C}_{15} \mathrm{H}_{14} \mathrm{O}_{6}$ & L-Epicatechin & $\begin{array}{l}289.0717 \\
{[\mathrm{M}-\mathrm{H}]^{-}}\end{array}$ & 7.333 & $\begin{array}{c}271.0235 \\
245.0411 \\
205.2713 \\
179.0110\end{array}$ & $\mathrm{~F}$ & \\
\hline 20 & 10.56 & $\mathrm{C}_{4} \mathrm{H}_{4} \mathrm{O}_{4}$ & Maleic acid & $\begin{array}{l}115.0036 \\
{[\mathrm{M}-\mathrm{H}]^{-}}\end{array}$ & -0.479 & 71.0124 & $\mathrm{PO}$ & \\
\hline 21 & 11.43 & $\mathrm{C}_{15} \mathrm{H}_{24} \mathrm{O}_{10}$ & Harpagide & $\begin{array}{l}363.1296 \\
{[\mathrm{M}-\mathrm{H}]^{-}}\end{array}$ & 0.977 & $\begin{array}{c}183.0652 \\
89.0228\end{array}$ & I & \\
\hline 22 & 12.53 & $\mathrm{C}_{16} \mathrm{H}_{18} \mathrm{O}_{9}$ & Chlorogenic acid & $\begin{array}{l}353.0878 \\
{[\mathrm{M}-\mathrm{H}]^{-}}\end{array}$ & 0.854 & $\begin{array}{c}191.0554 \\
179.0341 \\
173.0446 \\
161.0234 \\
155.0338 \\
137.0322 \\
135.0440 \\
93.0333\end{array}$ & $\mathrm{P}$ & Yes \\
\hline 23 & 14.13 & $\mathrm{C}_{8} \mathrm{H}_{8} \mathrm{O}_{4}$ & Methyl protocatechuic acid & $\begin{array}{l}167.0349 \\
{[\mathrm{M}-\mathrm{H}]^{-}}\end{array}$ & 0.508 & $\begin{array}{c}152.0106 \\
123.0439 \\
108.0203\end{array}$ & $\mathrm{PO}$ & \\
\hline 24 & 15.84 & $\mathrm{C}_{8} \mathrm{H}_{8} \mathrm{O}_{4}$ & Isovanillic acid & $\begin{array}{l}167.0349 \\
{[\mathrm{M}-\mathrm{H}]^{-}}\end{array}$ & -2.536 & 123.0439 & $\mathrm{PO}$ & \\
\hline 25 & 18.23 & $\mathrm{C}_{13} \mathrm{H}_{16} \mathrm{O}_{9}$ & Protocatechuic acid-4-glucoside & $\begin{array}{c}315.0721 \\
{[\mathrm{M}-\mathrm{H}]^{-}}\end{array}$ & -1.392 & 108.0204 & $\mathrm{PO}$ & \\
\hline
\end{tabular}


TABle 1: Continued.

\begin{tabular}{|c|c|c|c|c|c|c|c|c|}
\hline No. & $\begin{array}{c}\mathrm{tR} \\
(\mathrm{min})\end{array}$ & Formula & Identification & $\begin{array}{l}\text { Precursor } \\
\text { ions }(\mathrm{m} / \mathrm{z})\end{array}$ & $\begin{array}{c}\text { Diff } \\
(\mathrm{ppm})\end{array}$ & $\begin{array}{c}\text { Fragment } \\
(\mathrm{m} / \mathrm{z})\end{array}$ & Type & $\begin{array}{c}\text { Reference } \\
\text { standard }\end{array}$ \\
\hline 26 & 19.56 & $\mathrm{C}_{14} \mathrm{H}_{18} \mathrm{O}_{9}$ & 4-Glucopyranoxy-3-benzoic acid & $\begin{array}{l}329.0878 \\
{[\mathrm{M}-\mathrm{H}]^{-}}\end{array}$ & 4.340 & $\begin{array}{l}167.0340 \\
152.0105 \\
123.0439 \\
108.0204\end{array}$ & $\mathrm{O}$ & \\
\hline 27 & 20.23 & $\mathrm{C}_{9} \mathrm{H}_{12} \mathrm{O}_{5}$ & Rehmaglutin C & $\begin{array}{l}199.0611 \\
{[\mathrm{M}-\mathrm{H}]^{-}}\end{array}$ & 3.316 & $\begin{array}{l}155.0704 \\
137.0596\end{array}$ & I & \\
\hline 28 & 20.39 & $\mathrm{C}_{18} \mathrm{H}_{24} \mathrm{O}_{12}$ & Asperulosidic acid & $\begin{array}{l}431.1194 \\
{[\mathrm{M}-\mathrm{H}]^{-}}\end{array}$ & -3.114 & $\begin{array}{l}269.0198 \\
251.0098\end{array}$ & I & \\
\hline 29 & 21.77 & $\mathrm{C}_{16} \mathrm{H}_{18} \mathrm{O}_{9}$ & Neochlorogenic acid & $\begin{array}{l}353.0878 \\
{[\mathrm{M}-\mathrm{H}]^{-}}\end{array}$ & -0.699 & $\begin{array}{l}191.0554 \\
179.0341 \\
135.0440\end{array}$ & $\mathrm{P}$ & \\
\hline 30 & 22.36 & $\mathrm{C}_{16} \mathrm{H}_{18} \mathrm{O}_{9}$ & 4-Caffeoylquinic acid & $\begin{array}{l}353.0878 \\
{[\mathrm{M}-\mathrm{H}]^{-}}\end{array}$ & 3.941 & $\begin{array}{l}191.0554 \\
179.0340 \\
135.1440\end{array}$ & $\mathrm{P}$ & \\
\hline 31 & 23.28 & $\mathrm{C}_{20} \mathrm{H}_{24} \mathrm{O}_{7}$ & Cycloolivil & $\begin{array}{l}375.1449 \\
{[\mathrm{M}-\mathrm{H}]^{-}}\end{array}$ & -2.075 & $\begin{array}{l}327.1343 \\
297.1207 \\
257.1132 \\
151.0752\end{array}$ & $\mathrm{~L}$ & \\
\hline 32 & 24.28 & $\mathrm{C}_{9} \mathrm{H}_{8} \mathrm{O}_{4}$ & Caffeic acid & $\begin{array}{l}179.0349 \\
{[\mathrm{M}-\mathrm{H}]^{-}}\end{array}$ & 0.856 & 135.0440 & $\mathrm{P}$ & Yes \\
\hline 33 & 24.36 & $\mathrm{C}_{9} \mathrm{H}_{10} \mathrm{O}_{4}$ & Dihydrocaffeic acid & $\begin{array}{l}181.0506 \\
{[\mathrm{M}-\mathrm{H}]^{-}}\end{array}$ & 1.849 & $\begin{array}{l}163.0390 \\
135.0441 \\
119.0488\end{array}$ & $\mathrm{P}$ & \\
\hline 34 & 26.50 & $\mathrm{C}_{7} \mathrm{H}_{6} \mathrm{O}_{4}$ & Gentianic acid & $\begin{array}{l}153.0193 \\
{[\mathrm{M}-\mathrm{H}]^{-}}\end{array}$ & -0.295 & 109.0282 & $\mathrm{PO}$ & \\
\hline 35 & 27.23 & $\mathrm{C}_{7} \mathrm{H}_{6} \mathrm{O}_{4}$ & Protocatechuic acid & $\begin{array}{l}153.0193 \\
{[\mathrm{M}-\mathrm{H}]^{-}}\end{array}$ & 0.685 & $\begin{array}{l}\text { 109.0283 } \\
91.0175\end{array}$ & $\mathrm{PO}$ & \\
\hline 36 & 28.03 & $\mathrm{C}_{42} \mathrm{H}_{70} \mathrm{O}_{12}$ & Ginsenoside $\mathrm{F}_{4}$ & $\begin{array}{c}789.4759 \\
{[\mathrm{M}+\mathrm{Na}]^{+}}\end{array}$ & -1.837 & $\begin{array}{l}707.1499 \\
643.4222 \\
349.1090\end{array}$ & $\mathrm{~T}$ & \\
\hline 37 & 28.55 & $\mathrm{C}_{20} \mathrm{H}_{24} \mathrm{O}_{7}$ & Oleoresin & $\begin{array}{l}375.1449 \\
{[\mathrm{M}-\mathrm{H}]^{-}}\end{array}$ & -1.018 & $\begin{array}{l}179.0341 \\
161.0233\end{array}$ & $\mathrm{~L}$ & \\
\hline 38 & 29.40 & $\mathrm{C}_{16} \mathrm{H}_{18} \mathrm{O}_{9}$ & Cryptochlorogenic acid & $\begin{array}{l}353.0878 \\
{[\mathrm{M}-\mathrm{H}]^{-}}\end{array}$ & 3.516 & $\begin{array}{l}191.0554 \\
179.0340 \\
173.0446 \\
135.0440\end{array}$ & $\mathrm{P}$ & \\
\hline 39 & 30.90 & $\mathrm{C}_{20} \mathrm{H}_{24} 0_{7}$ & Olivil & $\begin{array}{l}375.1449 \\
{[\mathrm{M}-\mathrm{H}]^{-}}\end{array}$ & -2.635 & $\begin{array}{l}327.1360 \\
195.1251 \\
179.0341 \\
161.0220\end{array}$ & $\mathrm{~L}$ & \\
\hline 40 & 32.26 & $\mathrm{C}_{17} \mathrm{H}_{24} \mathrm{O}_{10}$ & Geniposide & $\begin{array}{l}387.1296 \\
{[\mathrm{M}-\mathrm{H}]^{-}}\end{array}$ & 3.128 & $\begin{array}{c}207.1025 \\
123.0444 \\
101.0232\end{array}$ & I & \\
\hline 41 & 33.49 & $\mathrm{C}_{33} \mathrm{H}_{44} \mathrm{O}_{19}$ & Naringin dihydrochalcone $4-\mathrm{O}-\beta$-D-glucoside & $\begin{array}{l}743.2404 \\
{[\mathrm{M}-\mathrm{H}]^{-}}\end{array}$ & 3.128 & $\begin{array}{c}373.1295 \\
313.1088 \\
181.0498 \\
151.0396\end{array}$ & $\mathrm{~F}$ & \\
\hline 42 & 34.26 & $\mathrm{C}_{27} \mathrm{H}_{32} \mathrm{O}_{16}$ & Hydroxysafflor yellow A & $\begin{array}{l}611.1617 \\
{[\mathrm{M}-\mathrm{H}]^{-}}\end{array}$ & 2.207 & $\begin{array}{l}491.1200 \\
473.1092 \\
403.1042 \\
325.0720\end{array}$ & $\mathrm{~F}$ & Yes \\
\hline 43 & 37.58 & $\mathrm{C}_{10} \mathrm{H}_{10} \mathrm{O}_{4}$ & Ferulic acid & $\begin{array}{l}193.0506 \\
{[\mathrm{M}-\mathrm{H}]^{-}}\end{array}$ & 2.208 & $\begin{array}{c}178.0264 \\
149.0598 \\
134.0362\end{array}$ & $\mathrm{P}$ & Yes \\
\hline 44 & 38.11 & $\mathrm{C}_{32} \mathrm{H}_{42} \mathrm{O}_{16}$ & Pinoresinol diglucoside & $\begin{array}{l}681.2400 \\
{[\mathrm{M}-\mathrm{H}]^{-}}\end{array}$ & 2.039 & $\begin{array}{l}519.5070 \\
357.1346 \\
151.0390 \\
136.0159\end{array}$ & $\mathrm{~L}$ & \\
\hline 45 & 39.59 & $\mathrm{C}_{16} \mathrm{H}_{18} \mathrm{O}_{8}$ & 3-O-p-Coumaroylquinic acid & $\begin{array}{l}337.0928 \\
{[\mathrm{M}-\mathrm{H}]^{-}}\end{array}$ & 6.688 & $\begin{array}{l}191.0553 \\
173.0448 \\
163.0390\end{array}$ & $\mathrm{P}$ & \\
\hline
\end{tabular}


TABle 1: Continued.

\begin{tabular}{|c|c|c|c|c|c|c|c|c|}
\hline No. & $\begin{array}{c}\mathrm{tR} \\
(\mathrm{min})\end{array}$ & Formula & Identification & $\begin{array}{l}\text { Precursor } \\
\text { ions }(\mathrm{m} / \mathrm{z})\end{array}$ & $\begin{array}{c}\text { Diff } \\
(\mathrm{ppm})\end{array}$ & $\begin{array}{c}\text { Fragment } \\
(\mathrm{m} / \mathrm{z})\end{array}$ & Type & $\begin{array}{c}\text { Reference } \\
\text { standard }\end{array}$ \\
\hline 46 & 40.21 & $\mathrm{C}_{27} \mathrm{H}_{36} \mathrm{O}_{13}$ & Citrusin B & $\begin{array}{l}567.2083 \\
{[\mathrm{M}-\mathrm{H}]^{-}}\end{array}$ & -4.289 & $\begin{array}{l}341.1384 \\
329.1394\end{array}$ & $\mathrm{~L}$ & \\
\hline 47 & 41.77 & $\mathrm{C}_{27} \mathrm{H}_{30} \mathrm{O}_{17}$ & Quercetin-3, 4'-O-di- $\beta$-glucopyranoside & $\begin{array}{l}625.1410 \\
{[\mathrm{M}-\mathrm{H}]^{-}}\end{array}$ & 0.831 & $\begin{array}{l}463.0884 \\
301.0350 \\
271.0243\end{array}$ & $\mathrm{~F}$ & \\
\hline 48 & 42.23 & $\mathrm{C}_{15} \mathrm{H}_{26} \mathrm{O}_{9}$ & Eucommioside & $\begin{array}{l}349.1504 \\
{[\mathrm{M}-\mathrm{H}]^{-}}\end{array}$ & -1.102 & $\begin{array}{l}187.1528 \\
89.0230\end{array}$ & I & \\
\hline 49 & 43.69 & $\mathrm{C}_{10} \mathrm{H}_{10} \mathrm{O}_{3}$ & Coniferyl aldehyde & $\begin{array}{l}177.0557 \\
{[\mathrm{M}-\mathrm{H}]^{-}}\end{array}$ & -0.101 & 162.0312 & $\mathrm{P}$ & \\
\hline 50 & 46.23 & $\mathrm{C}_{20} \mathrm{H}_{22} \mathrm{O}_{7}$ & Erythroglycerin- $\beta$-terpineol aldehyde ether & $\begin{array}{l}373.1292 \\
{[\mathrm{M}-\mathrm{H}]^{-}}\end{array}$ & 4.259 & $\begin{array}{l}177.0548 \\
165.0547 \\
150.0308\end{array}$ & $\mathrm{P}$ & \\
\hline 51 & 48.38 & $\mathrm{C}_{26} \mathrm{H}_{28} \mathrm{O}_{16}$ & Quercetin 3-O-sambubioside & $\begin{array}{l}595.1305 \\
{[\mathrm{M}+\mathrm{H}]^{+}}\end{array}$ & -0.638 & 301.0327 & $\mathrm{~F}$ & \\
\hline 52 & 49.62 & $\mathrm{C}_{26} \mathrm{H}_{32} \mathrm{O}_{11}$ & Pinoresinol-4'-O- $\beta$-D-glucopyranoside & $\begin{array}{l}519.1871 \\
{[\mathrm{M}-\mathrm{H}]^{-}}\end{array}$ & -3.929 & $\begin{array}{l}357.1345 \\
151.0390\end{array}$ & $\mathrm{~L}$ & \\
\hline 53 & 54.63 & $\mathrm{C}_{26} \mathrm{H}_{32} \mathrm{O}_{11}$ & Pinoresinol- $\beta$-D-glucoside & $\begin{array}{l}519.1871 \\
{[\mathrm{M}-\mathrm{H}]^{-}}\end{array}$ & 3.334 & $\begin{array}{l}357.1345 \\
342.1107 \\
311.1293 \\
151.0390 \\
136.0154\end{array}$ & $\mathrm{~L}$ & \\
\hline 54 & 55.16 & $\mathrm{C}_{21} \mathrm{H}_{20} \mathrm{O}_{12}$ & Isoquercitrin & $\begin{array}{l}463.0881 \\
{[\mathrm{M}-\mathrm{H}]^{-}}\end{array}$ & 3.623 & $\begin{array}{l}301.0349 \\
271.0321 \\
255.0299\end{array}$ & $\mathrm{~F}$ & Yes \\
\hline 55 & 56.44 & $\mathrm{C}_{22} \mathrm{H}_{28} \mathrm{O}_{14}$ & 5-( $3^{\prime}$-o-caffeoylglucosyl) quinine & $\begin{array}{l}515.1406 \\
{[\mathrm{M}-\mathrm{H}]^{-}}\end{array}$ & 8.420 & $\begin{array}{l}191.0555 \\
161.0234 \\
135.0440\end{array}$ & $\mathrm{P}$ & \\
\hline 56 & 57.45 & $\mathrm{C}_{22} \mathrm{H}_{28} \mathrm{O}_{14}$ & 1-O-(3 '-o-caffeoylglucosyl) quinine & $\begin{array}{l}515.1406 \\
{[\mathrm{M}-\mathrm{H}]^{-}}\end{array}$ & 2.499 & $\begin{array}{l}179.0341 \\
173.0446 \\
161.0233 \\
135.0440\end{array}$ & $\mathrm{P}$ & \\
\hline $57^{*}$ & 57.73 & $\mathrm{C}_{33} \mathrm{H}_{40} \mathrm{O}_{21}$ & $\begin{array}{l}\text { Quercetin 3-glucosyl-(1->3)-rhamnosyl-(1->6)- } \\
\text { galactoside }\end{array}$ & $\begin{array}{l}771.1989 \\
{[\mathrm{M}-\mathrm{H}]^{-}}\end{array}$ & 2.070 & $\begin{array}{l}609.1469 \\
463.0873 \\
301.0351\end{array}$ & $\mathrm{~F}$ & \\
\hline 58 & 58.66 & $\mathrm{C}_{28} \mathrm{H}_{36} \mathrm{O}_{13}$ & Syringaresionl-O- $\beta$-D-g1ucopyranoside & $\begin{array}{l}579.2083 \\
{[\mathrm{M}-\mathrm{H}]^{-}}\end{array}$ & 3.298 & 417.1557 & $\mathrm{P}$ & \\
\hline 59 & 59.50 & $\mathrm{C}_{35} \mathrm{H}_{60} \mathrm{O}_{6}$ & Daucosterol & $\begin{array}{l}575.4317 \\
{[\mathrm{M}-\mathrm{H}]^{-}}\end{array}$ & 2.329 & 397.7564 & $\mathrm{~T}$ & \\
\hline 60 & 62.59 & $\mathrm{C}_{25} \mathrm{H}_{24} \mathrm{O}_{12}$ & 1,5-Dicaffeoylquinic acid & $\begin{array}{l}515.1194 \\
{[\mathrm{M}-\mathrm{H}]^{-}}\end{array}$ & 2.499 & $\begin{array}{l}353.0881 \\
191.0554 \\
135.0440\end{array}$ & $\mathrm{P}$ & \\
\hline 61 & 63.34 & $\mathrm{C}_{25} \mathrm{H}_{24} \mathrm{O}_{12}$ & Isochlorogenic acid $\mathrm{A}$ & $\begin{array}{l}515.1194 \\
{[\mathrm{M}-\mathrm{H}]^{-}}\end{array}$ & 1.288 & $\begin{array}{l}353.0881 \\
191.0554 \\
179.0341 \\
173.0446 \\
135.0440\end{array}$ & $\mathrm{P}$ & \\
\hline 62 & 64.00 & $\mathrm{C}_{42} \mathrm{H}_{72} \mathrm{O}_{15}$ & $\begin{array}{c}\text { 6-O- } \beta \text {-D-Glucopyranosyl-20-o- } \beta \text {-D-glucopyranosyl- } \\
3 \beta, 6 \beta, 12 \beta, 20 \text { (S)7-25-pentaphydroxydammar-23- } \\
\text { enedroginsenoside } \mathrm{Rg}_{1}\end{array}$ & $\begin{array}{c}839.4763 \\
{[\mathrm{M}+\mathrm{Na}]^{+}}\end{array}$ & -2.644 & 659.4114 & $\mathrm{~T}$ & \\
\hline 63 & 65.10 & $\mathrm{C}_{25} \mathrm{H}_{24} \mathrm{O}_{12}$ & Isochlorogenic acid B & $\begin{array}{l}515.1194 \\
{[\mathrm{M}-\mathrm{H}]^{-}}\end{array}$ & 1.288 & $\begin{array}{l}353.0881 \\
335.0777 \\
191.0554 \\
179.0341 \\
173.0446\end{array}$ & $\mathrm{P}$ & \\
\hline 64 & 66.70 & $\mathrm{C}_{9} \mathrm{H}_{16} \mathrm{O}_{4}$ & Eucommitol & $\begin{array}{l}187.0975 \\
{[\mathrm{M}-\mathrm{H}]^{-}}\end{array}$ & 1.521 & $\begin{array}{l}169.0861 \\
143.1068 \\
125.0960\end{array}$ & I & Yes \\
\hline 65 & 66.72 & $\mathrm{C}_{6} \mathrm{H}_{4} \mathrm{O}_{4}$ & Coumalic acid & $\begin{array}{l}139.0036 \\
{[\mathrm{M}-\mathrm{H}]^{-}}\end{array}$ & 6.332 & 119.5097 & $\mathrm{O}$ & \\
\hline
\end{tabular}


TABle 1: Continued.

\begin{tabular}{|c|c|c|c|c|c|c|c|c|}
\hline No. & $\begin{array}{c}\mathrm{tR} \\
(\mathrm{min})\end{array}$ & Formula & Identification & $\begin{array}{l}\text { Precursor } \\
\text { ions }(\mathrm{m} / \mathrm{z})\end{array}$ & $\begin{array}{c}\text { Diff } \\
(\mathrm{ppm})\end{array}$ & $\begin{array}{l}\text { Fragment } \\
(m / z)\end{array}$ & Type & $\begin{array}{l}\text { Reference } \\
\text { standard }\end{array}$ \\
\hline 66 & 67.26 & $\mathrm{C}_{18} \mathrm{H}_{16} \mathrm{O}_{5}$ & Sideroxylin & $\begin{array}{l}311.0924 \\
{[\mathrm{M}-\mathrm{H}]^{-}}\end{array}$ & 2.177 & 267.0663 & $\mathrm{~F}$ & \\
\hline 67 & 67.68 & $\mathrm{C}_{21} \mathrm{H}_{20} \mathrm{O}_{12}$ & Hyperoside & $\begin{array}{l}463.0882 \\
{[\mathrm{M}-\mathrm{H}]^{-}}\end{array}$ & 4.206 & $\begin{array}{l}301.03455 \\
151.00258\end{array}$ & $\mathrm{~F}$ & \\
\hline 68 & 68.78 & $\mathrm{C}_{15} \mathrm{H}_{26} \mathrm{O}_{7}$ & $\begin{array}{l}\text { 2-(5-Hydroxyethyl-2,3-dimethyl-2-cyclopenten-1- } \\
\text { yl)-glucopyranoside }\end{array}$ & $\begin{array}{l}317.1605 \\
{[\mathrm{M}-\mathrm{H}]^{-}}\end{array}$ & 4.580 & $\begin{array}{l}243.1238 \\
225.1132\end{array}$ & I & \\
\hline 69 & 69.40 & $\mathrm{C}_{21} \mathrm{H}_{20} \mathrm{O}_{10}$ & Apigenin-7-O-glucuronide & $\begin{array}{l}431.0983 \\
{[\mathrm{M}-\mathrm{H}]^{-}}\end{array}$ & 3.147 & 269.0376 & $\mathrm{~F}$ & \\
\hline 70 & 70.29 & $\mathrm{C}_{9} \mathrm{H}_{16} \mathrm{O}_{3}$ & 1-Deoxyeucommitol & $\begin{array}{l}171.1026 \\
{[\mathrm{M}-\mathrm{H}]^{-}}\end{array}$ & 0.930 & $\begin{array}{l}127.1118 \\
125.0959\end{array}$ & I & \\
\hline 71 & 72.63 & $\mathrm{C}_{21} \mathrm{H}_{20} \mathrm{O}_{11}$ & Astragalin & $\begin{array}{l}447.0933 \\
{[\mathrm{M}-\mathrm{H}]^{-}}\end{array}$ & 2.532 & $\begin{array}{l}285.0395 \\
241.0829 \\
217.0886\end{array}$ & $\mathrm{~F}$ & \\
\hline 72 & 73.55 & $\mathrm{C}_{21} \mathrm{H}_{18} \mathrm{O}_{11}$ & Baicalin & $\begin{array}{l}445.0776 \\
{[\mathrm{M}-\mathrm{H}]^{-}}\end{array}$ & 4.724 & 269.0456 & $\mathrm{~F}$ & \\
\hline 73 & 74.23 & $\mathrm{C}_{27} \mathrm{H}_{30} \mathrm{O}_{15}$ & Nicotiflorin & $\begin{array}{l}593.1511 \\
{[\mathrm{M}-\mathrm{H}]^{-}}\end{array}$ & 3.681 & $\begin{array}{l}285.0404 \\
255.0307 \\
227.0352\end{array}$ & $\mathrm{~F}$ & \\
\hline 74 & 74.88 & $\mathrm{C}_{27} \mathrm{H}_{30} \mathrm{H}_{15}$ & Safflor yellow (A) & $\begin{array}{l}593.1511 \\
{[\mathrm{M}-\mathrm{H}]^{-}}\end{array}$ & 3.884 & 285.0404 & $\mathrm{~F}$ & Yes \\
\hline 75 & 75.61 & $\mathrm{C}_{18} \mathrm{H}_{14} \mathrm{O}_{6}$ & Milletenin C & $\begin{array}{l}325.0717 \\
{[\mathrm{M}-\mathrm{H}]^{-}}\end{array}$ & 2.650 & 310.0848 & $\mathrm{~F}$ & \\
\hline 76 & 76.26 & $\mathrm{C}_{12} \mathrm{H}_{16} \mathrm{O}_{3}$ & $\begin{array}{l}\text { 3-Butyl-4-hydroxy-4,5-dihydro-2-benzofuran- } \\
\text { 1(3H)-one }\end{array}$ & $\begin{array}{l}207.1026 \\
{[\mathrm{M}-\mathrm{H}]^{-}}\end{array}$ & 0.368 & 135.0443 & $\mathrm{O}$ & \\
\hline 77 & 77.03 & $\mathrm{C}_{11} \mathrm{H}_{12} \mathrm{O}_{4}$ & Ethyl caffeate & $\begin{array}{l}207.0662 \\
{[\mathrm{M}-\mathrm{H}]^{-}}\end{array}$ & 3.162 & $\begin{array}{l}179.0341 \\
161.0234 \\
135.0440\end{array}$ & $\mathrm{P}$ & \\
\hline 78 & 77.96 & $\mathrm{C}_{48} \mathrm{H}_{82} \mathrm{O}_{19}$ & Notoginsenoside $\mathrm{R}_{6}$ & $\begin{array}{c}985.5342 \\
{[\mathrm{M}+\mathrm{Na}]^{+}}\end{array}$ & -2.049 & $\begin{array}{l}365.1045 \\
305.0816\end{array}$ & $\mathrm{~T}$ & \\
\hline 79 & 78.56 & $\mathrm{C}_{48} \mathrm{H}_{82} \mathrm{O}_{19}$ & Notoginsenoside $R_{3}$ & $\begin{array}{c}985.5342 \\
{[\mathrm{M}+\mathrm{Na}]^{+}}\end{array}$ & 2.402 & $\begin{array}{l}645.4159 \\
365.1044\end{array}$ & $\mathrm{~T}$ & \\
\hline $80^{*}$ & 79.20 & $\mathrm{C}_{28} \mathrm{H}_{32} \mathrm{O}_{16}$ & 6-Methoxykaempferol 3-robinobioside & $\begin{array}{l}623.16175 \\
{[\mathrm{M}-\mathrm{H}]^{-}}\end{array}$ & 1.524 & $\begin{array}{l}315.0509 \\
301.0320 \\
300.0276\end{array}$ & $\mathrm{~F}$ & \\
\hline $81^{*}$ & 79.40 & $\mathrm{C}_{29} \mathrm{H}_{36} \mathrm{O}_{15}$ & $\begin{array}{l}\text { 3,4,6-Trihydroxy-4, } 2^{\prime} \text {-dimethoxychalcone } 4^{\prime} \text {-O- } \\
\text { rutinoside }\end{array}$ & $\begin{array}{l}623.19814 \\
{[\mathrm{M}-\mathrm{H}]^{-}}\end{array}$ & 6.18 & $\begin{array}{l}315.0510 \\
301.0313 \\
300.0376\end{array}$ & $\mathrm{~F}$ & \\
\hline 82 & 79.58 & $\mathrm{C}_{48} \mathrm{H}_{82} \mathrm{O}_{19}$ & Notoginsenoside $\mathrm{M}$ & $\begin{array}{c}985.5342 \\
{[\mathrm{M}+\mathrm{Na}]^{+}}\end{array}$ & -3.227 & $\begin{array}{l}805.4688 \\
365.1047\end{array}$ & $\mathrm{~T}$ & \\
\hline 83 & 81.76 & $\mathrm{C}_{48} \mathrm{H}_{82} \mathrm{O}_{19}$ & Notoginsenoside $\mathrm{N}$ & $\begin{array}{c}985.5342 \\
{[\mathrm{M}+\mathrm{Na}]^{+}}\end{array}$ & -2.983 & 805.4689 & $\mathrm{~T}$ & \\
\hline 84 & 82.77 & $\mathrm{C}_{48} \mathrm{H}_{82} \mathrm{O}_{19}$ & 20-O-Glucoginsenoside Rf & $\begin{array}{c}985.5342 \\
{[\mathrm{M}+\mathrm{Na}]^{+}}\end{array}$ & -1.684 & $\begin{array}{l}805.4689 \\
365.2320\end{array}$ & $\mathrm{~T}$ & \\
\hline $85^{*}$ & 83.83 & $\mathrm{C}_{23} \mathrm{H}_{22} \mathrm{O}_{11}$ & Apigenin $7-\left(2^{\prime \prime}\right.$-acetylglucoside $)$ & $\begin{array}{l}473.1089 \\
{[\mathrm{M}-\mathrm{H}]^{-}}\end{array}$ & 1.569 & $\begin{array}{l}413.0891 \\
269.0379\end{array}$ & $\mathrm{~F}$ & \\
\hline 86 & 84.34 & $\mathrm{C}_{41} \mathrm{H}_{68} \mathrm{O}_{12}$ & Notoginsenoside $\mathrm{T}_{5}$ & $\begin{array}{c}775.4602 \\
{[\mathrm{M}+\mathrm{Na}]^{+}}\end{array}$ & -2.385 & $\begin{array}{l}692.0035 \\
643.3312 \\
463.3556 \\
335.0930\end{array}$ & $\mathrm{~T}$ & \\
\hline 87 & 84.47 & $\mathrm{C}_{47} \mathrm{H}_{80} \mathrm{O}_{18}$ & Notoginsenoside $\mathrm{R}_{1}$ & $\begin{array}{l}931.5271 \\
{[\mathrm{M}-\mathrm{H}]^{-}}\end{array}$ & 0.633 & $\begin{array}{l}799.4888 \\
637.4328 \\
475.3800 \\
391.0658\end{array}$ & $\mathrm{~T}$ & Yes \\
\hline 88 & 85.37 & $\mathrm{C}_{15} \mathrm{H}_{10} \mathrm{O}_{6}$ & Luteolin & $\begin{array}{l}285.0404 \\
{[\mathrm{M}-\mathrm{H}]^{-}}\end{array}$ & 4.720 & $\begin{array}{c}257.0453 \\
151.0030\end{array}$ & $\mathrm{~F}$ & \\
\hline 89 & 85.76 & $\mathrm{C}_{42} \mathrm{H}_{72} \mathrm{O}_{14}$ & Majoroside $\mathrm{F}_{4}$ & $\begin{array}{c}823.4814 \\
{[\mathrm{M}+\mathrm{Na}]^{+}}\end{array}$ & -1.603 & 643.4166 & $\mathrm{~T}$ & \\
\hline 90 & 86.17 & $\mathrm{C}_{42} \mathrm{H}_{72} \mathrm{O}_{14}$ & $\begin{array}{l}\text { 3-O- } \beta \text {-D-Glucopyranosyl-6-O- } \beta \text {-D-glucopyranosyl- } \\
20-(\mathrm{S}) \text {-protopanaxatriol }\end{array}$ & $\begin{array}{c}823.4814 \\
{[\mathrm{M}+\mathrm{Na}]^{+}}\end{array}$ & -3.157 & $\begin{array}{l}703.0069 \\
643.4163\end{array}$ & $\mathrm{~T}$ & \\
\hline
\end{tabular}


TABle 1: Continued.

\begin{tabular}{|c|c|c|c|c|c|c|c|c|}
\hline No. & $\begin{array}{c}\mathrm{tR} \\
(\mathrm{min})\end{array}$ & Formula & Identification & $\begin{array}{l}\text { Precursor } \\
\text { ions }(m / z) \\
\end{array}$ & $\begin{array}{c}\text { Diff } \\
(\mathrm{ppm})\end{array}$ & $\begin{array}{c}\text { Fragment } \\
(\mathrm{m} / \mathrm{z})\end{array}$ & Type & $\begin{array}{c}\text { Reference } \\
\text { standard }\end{array}$ \\
\hline 91 & 87.18 & $\mathrm{C}_{42} \mathrm{H}_{72} \mathrm{O}_{14}$ & Gynoside B & $\begin{array}{c}823.4814 \\
{[\mathrm{M}+\mathrm{Na}]^{+}}\end{array}$ & -1.603 & 643.4164 & $\mathrm{~T}$ & \\
\hline 92 & 87.69 & $\mathrm{C}_{42} \mathrm{H}_{72} \mathrm{O}_{14}$ & Ginsenoside $\mathrm{Rg}_{1}$ & $\begin{array}{c}823.4814 \\
{[\mathrm{M}+\mathrm{Na}]^{+}}\end{array}$ & -1.603 & 643.4104 & $\mathrm{~T}$ & \\
\hline 93 & 88.21 & $\mathrm{C}_{45} \mathrm{H}_{74} \mathrm{O}_{17}$ & Malonyl ginsenoside $\mathrm{Rg}_{1}$ & $\begin{array}{c}909.4818 \\
{[\mathrm{M}+\mathrm{Na}]^{+}}\end{array}$ & -1.891 & $\begin{array}{l}865.4895 \\
729.4166 \\
685.4270\end{array}$ & $\mathrm{~T}$ & \\
\hline 94 & 89.59 & $\mathrm{C}_{48} \mathrm{H}_{80} \mathrm{O}_{19}$ & Notoginsenoside G & $\begin{array}{c}983.5186 \\
{[\mathrm{M}+\mathrm{Na}]^{+}}\end{array}$ & -2.897 & 803.4535 & $\mathrm{~T}$ & \\
\hline $95^{*}$ & 89.94 & $\mathrm{C}_{30} \mathrm{H}_{28} \mathrm{O}_{12}$ & $\begin{array}{l}4,2^{\prime}, 3^{\prime}, 4^{\prime}-\text { Tetrahydroxychalcone } 4^{\prime}-\mathrm{O}-\left(2^{\prime \prime}-\mathrm{O}-\mathrm{p}-\right. \\
\text { coumaroyl) glucoside }\end{array}$ & $\begin{array}{l}579.1507 \\
{[\mathrm{M}-\mathrm{H}]^{-}}\end{array}$ & 0.622 & $\begin{array}{l}271.0614 \\
151.0027 \\
107.0126\end{array}$ & $\mathrm{~F}$ & \\
\hline 96 & 90.05 & $\mathrm{C}_{15} \mathrm{H}_{10} \mathrm{O}_{5}$ & Genistein & $\begin{array}{l}269.0455 \\
{[\mathrm{M}-\mathrm{H}]^{-}}\end{array}$ & 4.572 & $\begin{array}{c}225.0554 \\
201.0555 \\
151.0027 \\
117.0329 \\
107.0124\end{array}$ & $\mathrm{~F}$ & Yes \\
\hline 97 & 90.57 & $\mathrm{C}_{15} \mathrm{H}_{10} \mathrm{O}_{5}$ & Apigenin & $\begin{array}{l}269.0455 \\
{[\mathrm{M}-\mathrm{H}]^{-}}\end{array}$ & 4.572 & $\begin{array}{c}225.0555 \\
201.0553 \\
151.0025 \\
117.0328 \\
107.0124\end{array}$ & $\mathrm{~F}$ & Yes \\
\hline 98 & 91.24 & $\mathrm{C}_{41} \mathrm{H}_{70} \mathrm{O}_{13}$ & Pseudoginsenoside $\mathrm{RT}_{3}$ & $\begin{array}{c}793.4708 \\
{[\mathrm{M}+\mathrm{Na}]^{+}}\end{array}$ & 3.882 & 613.4072 & $\mathrm{~T}$ & \\
\hline 99 & 91.79 & $\mathrm{C}_{44} \mathrm{H}_{74} \mathrm{O}_{15}$ & Yesanchinoside D & $\begin{array}{c}865.4919 \\
{[\mathrm{M}+\mathrm{Na}]^{+}}\end{array}$ & -4.633 & 685.4267 & $\mathrm{~T}$ & \\
\hline 100 & 92.47 & $\mathrm{C}_{30} \mathrm{H}_{26} \mathrm{O}_{12}$ & Apigenin-7-O-( $6^{\prime \prime}$-coumaroyl) glucoside & $\begin{array}{l}577.1351 \\
{[\mathrm{M}-\mathrm{H}]^{-}}\end{array}$ & 1.390 & $\begin{array}{l}431.0988 \\
269.0457\end{array}$ & $\mathrm{~F}$ & \\
\hline 101 & 92.96 & $\mathrm{C}_{20} \mathrm{H}_{22} \mathrm{O}_{6}$ & Epipinoresinol & $\begin{array}{l}357.1343 \\
{[\mathrm{M}-\mathrm{H}]^{-}}\end{array}$ & 1.216 & $\begin{array}{c}151.1533 \\
136.0809 \\
121.0282\end{array}$ & $\mathrm{~L}$ & \\
\hline 102 & 94.52 & $\mathrm{C}_{42} \mathrm{H}_{72} \mathrm{O}_{14}$ & Ginsenoside Rf & $\begin{array}{c}823.4814 \\
{[\mathrm{M}+\mathrm{Na}]^{+}}\end{array}$ & -2.113 & $\begin{array}{l}661.5368 \\
641.4468 \\
365.1043\end{array}$ & $\mathrm{~T}$ & \\
\hline 103 & 95.39 & $\mathrm{C}_{41} \mathrm{H}_{70} \mathrm{O}_{13}$ & Notoginsenoside $R_{2}$ & $\begin{array}{c}793.4708 \\
{[\mathrm{M}+\mathrm{Na}]^{+}}\end{array}$ & -2.583 & $\begin{array}{l}661.4249 \\
481.3630 \\
335.0939\end{array}$ & $\mathrm{~T}$ & \\
\hline 104 & 95.89 & $\mathrm{C}_{42} \mathrm{H}_{72} \mathrm{O}_{13}$ & Ginsenoside $\mathrm{Rg}_{2}$ & $\begin{array}{c}807.4865 \\
{[\mathrm{M}+\mathrm{Na}]^{+}}\end{array}$ & -1.362 & $\begin{array}{l}661.4281 \\
481.3676 \\
349.1101\end{array}$ & $\mathrm{~T}$ & \\
\hline 105 & 96.47 & $\mathrm{C}_{36} \mathrm{H}_{62} \mathrm{O}_{9}$ & Gypenoside LXXVI & $\begin{array}{c}661.4286 \\
{[\mathrm{M}+\mathrm{Na}]^{+}}\end{array}$ & 2.479 & $\begin{array}{l}601.2890 \\
481.3620\end{array}$ & $\mathrm{~T}$ & \\
\hline 106 & 97.28 & $\mathrm{C}_{36} \mathrm{H}_{62} \mathrm{O}_{9}$ & Ginsenoside $\mathrm{Rh}_{1}$ & $\begin{array}{c}661.4286 \\
{[\mathrm{M}+\mathrm{Na}]^{+}}\end{array}$ & -1.769 & $\begin{array}{l}481.3650 \\
413.2539\end{array}$ & $\mathrm{~T}$ & \\
\hline 107 & 98.42 & $\mathrm{C}_{59} \mathrm{H}_{100} \mathrm{O}_{27}$ & Ginsenoside $\mathrm{Ra}_{3}$ & $\begin{array}{l}1263.6344 \\
{[\mathrm{M}+\mathrm{Na}]^{+}}\end{array}$ & -2.327 & $\begin{array}{l}789.4784 \\
497.1457 \\
437.1239\end{array}$ & $\mathrm{~T}$ & \\
\hline 108 & 99.42 & $\mathrm{C}_{59} \mathrm{H}_{100} \mathrm{O}_{27}$ & Notoginsenoside Fa & $\begin{array}{l}1263.6344 \\
{[\mathrm{M}+\mathrm{Na}]^{+}}\end{array}$ & -0.688 & 921.5158 & $\mathrm{~T}$ & \\
\hline 109 & 100.51 & $\mathrm{C}_{54} \mathrm{H}_{92} \mathrm{O}_{22}$ & Notoginsenoside I & $\begin{array}{l}1115.5972 \\
{[\mathrm{M}+\mathrm{Na}]^{+}}\end{array}$ & -1.470 & $\begin{array}{l}773.4795 \\
365.1046\end{array}$ & $\mathrm{~T}$ & \\
\hline 110 & 101.91 & $\mathrm{C}_{54} \mathrm{H}_{92} \mathrm{O}_{23}$ & Ginsenoside $\mathrm{Rb}_{1}$ & $\begin{array}{l}1107.5956 \\
{[\mathrm{M}-\mathrm{H}]^{-}}\end{array}$ & 0.589 & $\begin{array}{c}945.5432 \\
783.4906 \\
621.4368 \\
459.3851\end{array}$ & $\mathrm{~T}$ & Yes \\
\hline 111 & 102.94 & $\mathrm{C}_{42} \mathrm{H}_{72} \mathrm{O}_{13}$ & Ginsenoside $\mathrm{Rg}_{3}$ & $\begin{array}{c}807.4865 \\
{[\mathrm{M}+\mathrm{Na}]^{+}}\end{array}$ & -0.904 & 365.1046 & $\mathrm{~T}$ & \\
\hline 112 & 103.85 & $\mathrm{C}_{48} \mathrm{H}_{82} \mathrm{O}_{18}$ & Ginsenoside Re & $\begin{array}{c}969.5393 \\
{[\mathrm{M}+\mathrm{Na}]^{+}}\end{array}$ & -1.908 & 789.4742 & $\mathrm{~T}$ & Yes \\
\hline 113 & 105.07 & $\mathrm{C}_{54} \mathrm{H}_{92} \mathrm{O}_{23}$ & Yesanchinoside E & $\begin{array}{l}1131.5921 \\
{[\mathrm{M}+\mathrm{Na}]^{+}}\end{array}$ & -5.768 & $\begin{array}{c}789.4737 \\
365.1045\end{array}$ & $\mathrm{~T}$ & \\
\hline
\end{tabular}


TABle 1: Continued.

\begin{tabular}{|c|c|c|c|c|c|c|c|c|}
\hline No. & $\begin{array}{c}\mathrm{tR} \\
(\mathrm{min}) \\
\end{array}$ & Formula & Identification & $\begin{array}{l}\text { Precursor } \\
\text { ions }(m / z) \\
\end{array}$ & $\begin{array}{c}\text { Diff } \\
(\mathrm{ppm})\end{array}$ & $\begin{array}{c}\text { Fragment } \\
(m / z)\end{array}$ & Type & $\begin{array}{c}\text { Reference } \\
\text { standard }\end{array}$ \\
\hline 114 & 106.08 & $\mathrm{C}_{38} \mathrm{H}_{64} \mathrm{O}_{10}$ & $6^{\prime}$-O-Acetylginsenoside $F_{1}$ & $\begin{array}{c}703.4391 \\
{[\mathrm{M}+\mathrm{Na}]^{+}}\end{array}$ & 0.071 & 481.3647 & $\mathrm{~T}$ & \\
\hline 115 & 106.58 & $\mathrm{C}_{56} \mathrm{H}_{94} \mathrm{O}_{24}$ & Quinquenoside $\mathrm{R}_{1}$ & $\begin{array}{l}1173.6027 \\
{[\mathrm{M}+\mathrm{Na}]^{+}}\end{array}$ & -2.548 & $\begin{array}{l}831.4845 \\
365.1044\end{array}$ & $\mathrm{~T}$ & \\
\hline 116 & 107.65 & $\mathrm{C}_{56} \mathrm{H}_{94} \mathrm{O}_{24}$ & $6^{\prime \prime \prime}$-O-Acetylginsenoside $\mathrm{Rb}_{1}$ & $\begin{array}{l}1173.6027 \\
{[\mathrm{M}+\mathrm{Na}]^{+}}\end{array}$ & -2.326 & $\begin{array}{l}831.4845 \\
789.4744 \\
407.1151 \\
347.0945\end{array}$ & $\mathrm{~T}$ & \\
\hline 117 & 108.75 & $\mathrm{C}_{53} \mathrm{H}_{90} \mathrm{O}_{22}$ & Ginsenoside $\mathrm{Rb}_{2}$ & $\begin{array}{l}1101.5815 \\
{[\mathrm{M}+\mathrm{Na}]^{+}}\end{array}$ & -1.479 & $\begin{array}{l}789.4740 \\
335.0939\end{array}$ & $\mathrm{~T}$ & \\
\hline 118 & 109.76 & $\mathrm{C}_{53} \mathrm{H}_{90} \mathrm{O}_{22}$ & Notoginsenoside L & $\begin{array}{l}1101.5815 \\
{[\mathrm{M}+\mathrm{Na}]^{+}}\end{array}$ & -1.479 & 789.4740 & $\mathrm{~T}$ & \\
\hline 119 & 110.33 & $\mathrm{C}_{57} \mathrm{H}_{94} \mathrm{O}_{26}$ & Malonyl ginsenoside $\mathrm{Rb}_{1}$ & $\begin{array}{l}1217.5925 \\
{[\mathrm{M}+\mathrm{Na}]^{+}}\end{array}$ & -2.727 & $\begin{array}{l}1173.5993 \\
875.4738 \\
831.4844 \\
789.4738\end{array}$ & $\mathrm{~T}$ & \\
\hline 120 & 111.31 & $\mathrm{C}_{48} \mathrm{H}_{82} \mathrm{O}_{17}$ & Vina-ginsenoside $R_{3}$ & $\begin{array}{c}953.5444 \\
{[\mathrm{M}+\mathrm{Na}]^{+}}\end{array}$ & -2.349 & 773.4788 & $\mathrm{~T}$ & \\
\hline 121 & 111.47 & $\mathrm{C}_{48} \mathrm{H}_{82} \mathrm{O}_{18}$ & Gypenoside XVII & $\begin{array}{c}969.5393 \\
{[\mathrm{M}+\mathrm{Na}]^{+}}\end{array}$ & -0.908 & 365.1048 & $\mathrm{~T}$ & \\
\hline 122 & 112.04 & $\mathrm{C}_{57} \mathrm{H}_{94} \mathrm{O}_{26}$ & $\begin{array}{c}\text { 3-( } \beta \text {-D-Glucopyranosyl- } \beta \text {-D-glucopyranosyl)-20-O- } \\
\text { (6-O-malonyl- } \beta \text {-D-glucopyranosyl- } \beta \text {-D- } \\
\text { glucopyranosyl })-3 \beta, 12 \beta, 20(\mathrm{~S}) \text {-trihydroxydammar- } \\
24 \text {-ene }\end{array}$ & $\begin{array}{l}1217.5925 \\
{[\mathrm{M}+\mathrm{Na}]^{+}}\end{array}$ & -1.07 & $\begin{array}{c}1173.6008 \\
1131.5912 \\
875.4739 \\
831.4839 \\
789.4733 \\
451.1044 \\
407.1150\end{array}$ & $\mathrm{~T}$ & \\
\hline 123 & 112.53 & $\mathrm{C}_{48} \mathrm{H}_{82} \mathrm{O}_{18}$ & Ginsenoside Rd & $\begin{array}{l}945.5428 \\
{[\mathrm{M}-\mathrm{H}]^{-}}\end{array}$ & 0.857 & $\begin{array}{l}783.4907 \\
621.4375 \\
459.3848 \\
375.3146\end{array}$ & $\mathrm{~T}$ & Yes \\
\hline 124 & 112.57 & $\mathrm{C}_{36} \mathrm{H}_{62} \mathrm{O}_{8}$ & Notoginsenoside $\mathrm{R}_{7}$ & $\begin{array}{c}645.4336 \\
{[\mathrm{M}+\mathrm{Na}]^{+}}\end{array}$ & -3.249 & $\begin{array}{l}627.3813 \\
465.3691\end{array}$ & $\mathrm{~T}$ & \\
\hline 125 & 113.48 & $\begin{array}{l}\mathrm{C}_{36} \mathrm{H}_{60} \mathrm{O}_{8} \\
\mathrm{C}_{36} \mathrm{H}_{60} \mathrm{O}_{7}\end{array}$ & Ginsenoside $\mathrm{Rh}_{3}$ & $\begin{array}{c}643.4180 \\
{[\mathrm{M}+\mathrm{Na}]^{+}}\end{array}$ & -3.232 & $\begin{array}{c}583.3644 \\
463.3514\end{array}$ & $\mathrm{~T}$ & \\
\hline 126 & 113.89 & $\mathrm{C}_{51} \mathrm{H}_{84} \mathrm{O}_{21}$ & Malonyl ginsenoside Rd & $\begin{array}{l}1055.5397 \\
{[\mathrm{M}+\mathrm{Na}]^{+}}\end{array}$ & 3.598 & $\begin{array}{l}875.4738 \\
789.4740\end{array}$ & $\mathrm{~T}$ & \\
\hline 127 & 114.07 & $\mathrm{C}_{48} \mathrm{H}_{82} \mathrm{O}_{18}$ & Gypenoside LXXII & $\begin{array}{c}969.5393 \\
{[\mathrm{M}+\mathrm{Na}]^{+}}\end{array}$ & -1.691 & 789.4739 & $\mathrm{~T}$ & \\
\hline 128 & 114.88 & $\mathrm{C}_{36} \mathrm{H}_{62} \mathrm{O}_{11}$ & Notoginsenoside $T_{4}$ & $\begin{array}{c}693.4184 \\
{[\mathrm{M}+\mathrm{Na}]^{+}}\end{array}$ & -3.763 & 633.3707 & $\mathrm{~T}$ & \\
\hline 129 & 115.36 & $\mathrm{C}_{47} \mathrm{H}_{80} \mathrm{O}_{17}$ & $\begin{array}{l}\text { 3-O-[ } \beta \text {-D-Glucopyranosyl(1-2)- } \beta \text {-D- } \\
\text { glucopyranosyl]-20-O- } \beta \text {-D-xylopyranosyl- } \\
3 \beta, 12 \beta, 20(\mathrm{~s}) \text {-trihydroxydammar-24-ene }\end{array}$ & $\begin{array}{c}939.5287 \\
{[\mathrm{M}+\mathrm{Na}]^{+}}\end{array}$ & -0.872 & 789.4735 & $\mathrm{~T}$ & \\
\hline 130 & 116.22 & $\mathrm{C}_{15} \mathrm{H}_{10} \mathrm{O}_{5}$ & Baicalein & $\begin{array}{l}269.0455 \\
{[\mathrm{M}-\mathrm{H}]^{-}}\end{array}$ & 4.238 & 197.1905 & $\mathrm{~F}$ & Yes \\
\hline 131 & 117.02 & $\mathrm{C}_{47} \mathrm{H}_{80} \mathrm{O}_{18}$ & $\begin{array}{l}\text { 6-O-[Xylopyranosyl- } \beta \text {-D-glucopyranosyl]- } \\
3 \beta, 6 \beta, 12 \beta, 20(\mathrm{~s}), 25 \text {-pentahydroxydammar }\end{array}$ & $\begin{array}{c}811.4814 \\
{[\mathrm{M}+\mathrm{Na}]^{+}}\end{array}$ & 1.208 & $\begin{array}{l}793.3365 \\
751.2600 \\
679.2239 \\
499.1350 \\
412.1227 \\
335.0018\end{array}$ & $\mathrm{~T}$ & \\
\hline 132 & 117.70 & $\mathrm{C}_{20} \mathrm{H}_{22} \mathrm{O}_{6}$ & Pinoresinol & $\begin{array}{l}357.1343 \\
{[\mathrm{M}-\mathrm{H}]^{-}}\end{array}$ & 1.340 & $\begin{array}{l}313.1811 \\
151.1520 \\
136.0819\end{array}$ & $\mathrm{~L}$ & \\
\hline 133 & 118.87 & $\mathrm{C}_{42} \mathrm{H}_{72} \mathrm{O}_{13}$ & Ginsenoside $\mathrm{F}_{2}$ & $\begin{array}{c}807.4865 \\
{[\mathrm{M}+\mathrm{Na}]^{+}}\end{array}$ & 0.458 & 627.4217 & $\mathrm{~T}$ & \\
\hline 134 & 120.08 & $\mathrm{C}_{42} \mathrm{H}_{72} \mathrm{O}_{13}$ & Gypenoside LXXV & $\begin{array}{c}807.4865 \\
{[\mathrm{M}+\mathrm{Na}]^{+}}\end{array}$ & 7.789 & 365.1045 & $\mathrm{~T}$ & \\
\hline
\end{tabular}


TABle 1: Continued.

\begin{tabular}{|c|c|c|c|c|c|c|c|c|}
\hline No. & $\begin{array}{c}\mathrm{tR} \\
(\mathrm{min})\end{array}$ & Formula & Identification & $\begin{array}{l}\text { Precursor } \\
\text { ions }(m / z)\end{array}$ & $\begin{array}{c}\text { Diff } \\
(\mathrm{ppm})\end{array}$ & $\begin{array}{c}\text { Fragment } \\
(\mathrm{m} / \mathrm{z})\end{array}$ & Type & $\begin{array}{c}\text { Reference } \\
\text { standard }\end{array}$ \\
\hline 135 & 121.53 & $\mathrm{C}_{29} \mathrm{H}_{42} \mathrm{O}_{5}$ & Ulmoidol & $\begin{array}{l}469.2959 \\
{[\mathrm{M}-\mathrm{H}]^{-}}\end{array}$ & 3.715 & 423.2238 & $\mathrm{~T}$ & \\
\hline 136 & 121.65 & $\mathrm{C}_{28} \mathrm{H}_{34} \mathrm{O}_{4}$ & Unknown & $\begin{array}{l}433.2384 \\
{[\mathrm{M}-\mathrm{H}]^{-}}\end{array}$ & -0.685 & 433.2577 & $\mathrm{O}$ & \\
\hline 137 & 122.04 & $\mathrm{C}_{36} \mathrm{H}_{60} \mathrm{O}_{9}$ & Ginsenoside $\mathrm{Rh}_{7}$ & $\begin{array}{c}659.4129 \\
{[\mathrm{M}+\mathrm{Na}]^{+}}\end{array}$ & -0.349 & 599.3925 & $\mathrm{~T}$ & \\
\hline 138 & 122.50 & $\mathrm{C}_{32} \mathrm{H}_{42} \mathrm{O}_{17}$ & $\begin{array}{l}\text { 1-Hydroxypinoresinol- } 4,4^{\prime \prime} \text {-di-O- } \beta \text {-D- } \\
\text { glucopyranoside }\end{array}$ & $\begin{array}{l}697.2349 \\
{[\mathrm{M}-\mathrm{H}]^{-}}\end{array}$ & 0.112 & $\begin{array}{l}535.1532 \\
373.0323\end{array}$ & $\mathrm{~L}$ & \\
\hline 139 & 122.99 & $\mathrm{C}_{20} \mathrm{H}_{24} \mathrm{O}_{8}$ & Threo-dihydroxydehy-drodiconiferyl alcohol & $\begin{array}{l}391.1398 \\
{[\mathrm{M}-\mathrm{H}]^{-}}\end{array}$ & -3.313 & $\begin{array}{l}313.1747 \\
295.0882\end{array}$ & $\mathrm{~L}$ & \\
\hline 140 & 123.91 & $\mathrm{C}_{16} \mathrm{H}_{32} \mathrm{O}_{2}$ & Palmitic acid & $\begin{array}{l}255.2329 \\
{[\mathrm{M}-\mathrm{H}]^{-}}\end{array}$ & 1.978 & 241.3251 & $\mathrm{O}$ & Yes \\
\hline 141 & 124.01 & $\mathrm{C}_{20} \mathrm{H}_{24} \mathrm{O}_{8}$ & Erytho-dihydroxydehydrodiconiferyl alcohol & $\begin{array}{l}391.1398 \\
{[\mathrm{M}-\mathrm{H}]^{-}}\end{array}$ & -2.359 & $\begin{array}{l}341.1587 \\
313.0930 \\
207.0832\end{array}$ & $\mathrm{~L}$ & \\
\hline 142 & 125.83 & $\mathrm{C}_{18} \mathrm{H}_{36} \mathrm{O}_{2}$ & Palmitic acid ethyl ester & $\begin{array}{l}283.2642 \\
{[\mathrm{M}-\mathrm{H}]^{-}}\end{array}$ & 3.859 & 89.0229 & $\mathrm{O}$ & \\
\hline 143 & 127.10 & $\mathrm{C}_{9} \mathrm{H}_{12} \mathrm{O}_{4}$ & Eucommidiol & $\begin{array}{l}183.0662 \\
{[\mathrm{M}-\mathrm{H}]^{-}}\end{array}$ & 0.268 & $\begin{array}{c}139.1124 \\
93.7235\end{array}$ & I & \\
\hline
\end{tabular}

945.5432, $\mathrm{m} / z$ 783.4906, $\mathrm{m} / z$ 621.4368, and $\mathrm{m} / \mathrm{z} 459.3851$. Compared with the standard, compound 110 was identified as ginsenoside $R b_{1}$; the possible cleavage pathways of ginsenoside $\mathrm{Rb}_{1}$ are shown in Figure S2-A. Similarly, $[\mathrm{M}-\mathrm{H}]^{-}$ at $m / z 945.5428$ (compound 123) was tentatively identified ginsenoside Rd; the main fragment ions were [M-H-glc $]^{-} \mathrm{m}$ / $z$ 783.4907, [M-H-glc-glc] ${ }^{-} m / z 621.4375$, and [M-H-glc-glc$\mathrm{glc}^{-} \mathrm{m} / z$ 459.3848 [26]. Compound 87 was filtered by characteristic ion $\mathrm{m} / z$ 475.38, which tentatively identified PPT type ginsenoside. In the secondary mass spectrum, fragment ions $m / z$ 799.4888, $m / z 637.4328, m / z$ 475.3800, and $m / z 391.0658$ were [M-H-xyl] $]^{-}$, [M-H-xyl-glc] $]^{-}$, [M-Hxyl-glc-glc $]^{-}$, and aglycon; the possible cleavage pathways of $[\mathrm{M}-\mathrm{H}]^{-}$are shown in Figure S2-B.

By using the FSCIF strategy, a total of eleven compounds $(107,112,113,116,117,118,119,122,126,127$, and 129) were detected by characteristic ion of $789.47 \mathrm{Da} \quad([\mathrm{M}+\mathrm{Na}-$ glcglc $^{6}$ malonyl $]^{+}$). The retention time of compound 122 was $112.04 \mathrm{~min}$; the fragment ions $m / z 451.1044$ and $m / z 789.4733$ were a pair of complementary ions $\left[g_{c g l c}{ }^{6} \text { malonyl }+\mathrm{Na}\right]^{+}$ and $\left[\mathrm{M}+\mathrm{Na} \text {-gicglc }{ }^{6} \text { malonyl }\right]^{+}$. In addition, the fragment ions were observed in $\mathrm{m} / z 1173.6008,1131.5912$, 875.4739, 831.4839, and 407.1150, which were assigned to $\left[\mathrm{M}+\mathrm{Na}-\mathrm{CO}_{2}\right]^{+},[\mathrm{M}+\mathrm{Na}-$ malonyl $]^{+}, \quad[\mathrm{M}+\mathrm{Na} \text {-glcglc }]^{+}, \quad\left[\mathrm{M}+\mathrm{Na}-\left(\text { glcglc }+\mathrm{CO}_{2}\right)^{+}\right]$, and $\left[\text { glcglc }{ }^{6} \text { malonyl }+\mathrm{Na}-\mathrm{CO}_{2}\right]^{+}$fragment ions. The possible cleavage pathways of compound 122 are shown in Figure S2-C.

Additionally, the sugar type and its junction position were concluded with the application of NLF strategy. The position of sugar fragments on the aglycon was relatively fixed (C3, C6, and C12), and the main types of sugars were glc $(162.02 \mathrm{Da})$, rha $(146.01 \mathrm{Da})$, and xyl $(132.02 \mathrm{Da})$; the linkage between sugars is mainly $1-2$ and 1-6. In this experiment, nineteen compounds $(62,82,83,84,89,90$, $91,92,93,94,98,99,102,105,106,120,124,125$, and 133) were detected by NLF with $162.02 \mathrm{Da}$. Compounds 36, 86,
103 , and 104 filtered by $146.01 \mathrm{Da}$ or $132.02 \mathrm{Da}$ were obtained. Compound $103\left(\mathrm{C}_{42} \mathrm{H}_{72} \mathrm{O}_{13}\right)[\mathrm{M}+\mathrm{Na}]^{+}$at $\mathrm{m} / z$ 793.4708 tentatively annotated notoginsenoside $\mathrm{R}_{2}$; the main fragment ions were $[\mathrm{M}-\mathrm{H}-\mathrm{xyl}]^{-} \mathrm{m} / z$ 661.4249, [M$\mathrm{H}$-xyl-glc] ${ }^{-} m / z$ 481.3630, and [M-H-xyl-glc-rha] ${ }^{-} \mathrm{m} / z$ 335.0939. Compound $104\left(\mathrm{C}_{42} \mathrm{H}_{72} \mathrm{O}_{13}\right)[\mathrm{M}+\mathrm{Na}]^{+}$at $m / z$ 807.4865 tentatively annotated ginsenoside $\mathrm{Rg}_{2}$. First, the ion at $m / z 661.4281$ was formed by the neutral loss of a rhamnose unit of the ion at $m / z$ 807.4865. Second, the ion at $m / z 481.3676$ was formed by the neutral loss of a glucose unit of the ion at $m / z 661.4281$. Finally, ion at $m / z 349.1101$ was formed by the neutral loss of a xylose unit of the ion at $m / z 481.3676$.

3.2.2. Identification of Flavonoids. Most flavonoid aglycones were derivatives of quercetin, kaempferol, and apigenin, so we set $301.03 \mathrm{Da}, 285.04 \mathrm{Da}$, and $269.04 \mathrm{Da}$ as characteristic ions templates for these components annotation, which contributes to the rapid annotate flavonoids. A total of nine compounds $(1,2,47,51,54,57,67,80$, and 81$)$ screened with 301.03 Da were found; compound 1 showed $[\mathrm{M}-\mathrm{H}]^{-}$at $\mathrm{m} / z$ 609.1461, which was tentatively identified as rutin; its important fragment ion was $301.03 \mathrm{Da}$ in secondary mass spectra, indicating the neutral loss of $308.11 \mathrm{Da}\left(\mathrm{C}_{12} \mathrm{H}_{20} \mathrm{O}_{9}\right)$. In addition, the occurrences of $m / z 283.0325, m / z 255.0292$, and $m / z 227.0321$ were a better proof of [M-H-C ${ }_{12} \mathrm{H}_{20} \mathrm{O}_{9^{-}}$ $\left.\mathrm{H}_{2} \mathrm{O}\right]^{-}$, [M-H-C $\left.{ }_{12} \mathrm{H}_{20} \mathrm{O}_{9}-\mathrm{H}_{2} \mathrm{O}-\mathrm{CO}\right]^{-}$, and [M-H-C ${ }_{12} \mathrm{H}_{20} \mathrm{O}_{9^{-}}$ $\left.\mathrm{H}_{2} \mathrm{O}-2 \mathrm{CO}\right]^{-}$, which were the main peak, appearing in second mass spectra (Figure S3-A). Moreover, compounds 57, 80, and 81 were annotated for the first time in MTBD. Compound 57 showed $[\mathrm{M}-\mathrm{H}]^{-}$at $m / z 771.1989$; the ion at $m / z$ 609.1469 was formed by the neutral loss of a glucose unit of the ion at $m / z 771.1989$. Besides, the ion at $\mathrm{m} / z 463.0873$ was formed by the neutral loss of an xylose unit of the ion at $\mathrm{m} / z$ 609.1469. Finally, ion at $m / z 301.0351$ was formed by the 
neutral loss of a glucose unit of the ion at $\mathrm{m} / z 463.0873$. Hence, compound 57 was tentatively annotated Quercetin 3glucosyl-(1->3)-rhamnosyl-(1->6)-galactoside.

A total of five compounds (12, 71, 73, 74, and 88) acquired with $285.04 \mathrm{Da}$ were found. Compound 12 showed $[\mathrm{M}-\mathrm{H}]^{-}$at $\mathrm{m} / z 285.0404, \mathrm{~m} / z 257.0453, \mathrm{~m} / z 239.1650, \mathrm{~m} / z 229.0322$, and $\mathrm{m} /$ $z 185.0420$, corresponding to $[\mathrm{M}-\mathrm{H}-\mathrm{CO}]^{-},\left[\mathrm{M}-\mathrm{H}-\mathrm{CO}-\mathrm{H}_{2} \mathrm{O}\right]^{-}$, $[\mathrm{M}-\mathrm{H}-2 \mathrm{CO}]^{-}$, and $\left[\mathrm{M}-\mathrm{H}-2 \mathrm{CO}-\mathrm{CO}_{2}\right)^{-}$, which contributed to the crack of $\mathrm{C} 2-\mathrm{C} 3$ and $\mathrm{C} 4-\mathrm{C} 10$. In addition, the fracture of $\mathrm{C} 4-\mathrm{C} 10$ bond can also lead to the removal of $\mathrm{C}_{2} \mathrm{H}_{2} \mathrm{O}(42.02 \mathrm{Da})$, which corresponded to $\mathrm{m} / \mathrm{z}$ 243.1601. Next, the removal of $\mathrm{CO}_{2}$ $(44.01 \mathrm{Da})$ results in the generation of $m / z 199.0395$. By using the FSCIF strategy, seven compounds $(69,72,85,96,97,100$, and 130) screened with $269.04 \mathrm{Da}$ were found. Compound 97 was tentatively identified as apigenin; a high abundance secondary mass spectrometer fragment ion $\mathrm{m} / z 225.0555$ was formed after $\mathrm{CO}_{2}(44.01 \mathrm{Da})$ loss, indicating that apigenin derivatives were easier to lose $\mathrm{CO}_{2}$. In addition, $m / z 269.0455$ lost one molecule, $\mathrm{C}_{3} \mathrm{O}_{2}(68.02 \mathrm{Da})$, resulting in $\mathrm{m} / z 201.0553$. Apigenin, which is a flavonoid with double bond on the six-membered ring, can also undergo ring opening reaction of $\mathrm{C}$ ring, resulting in fragment ions such as $m / z 151.0025, m / z 117.0328$, and $m / z 107.0124$. These structural changes were also reflected at a retro-DielsAlder (RDA) reaction [27]. Hence, the characteristic ion of RDA was set by $151.00 \mathrm{Da}$; compounds $1,2,41,67,88,95,96$, and 97 screened with $151.00 \mathrm{Da}$ were found. Compound 95 was annotated as $4,2^{\prime}, 3^{\prime}, 4^{\prime}$-tetrahydroxychalcone $4^{\prime}-\mathrm{O}-\left(2^{\prime \prime}-\mathrm{O}-\mathrm{p}\right.$-coumaroyl) glucoside $(m / z 579.1507)$, which was being reported from MTBD for the first time. Its molecular ion peak $\mathrm{m} / z$ 269.0455 at $[\mathrm{M}-\mathrm{H}]^{-}$was observed; the fragment ions $\mathrm{m} / \mathrm{z}$ $271.0614, m / z 151.0027$, and $m / z 107.0126$ proved $[\mathrm{M}-\mathrm{H}-$ $\left.\mathrm{C}_{15} \mathrm{H}_{16} \mathrm{O}_{7}\right]^{-}$, [M-H-C $\left.{ }_{15} \mathrm{H}_{16} \mathrm{O}_{7}-\mathrm{C}_{8} \mathrm{H}_{8} \mathrm{O}\right]^{-}$, and [M-H-C ${ }_{15} \mathrm{H}_{16} \mathrm{O}_{7^{-}}$ $\left.\mathrm{C}_{8} \mathrm{H}_{8} \mathrm{O}-\mathrm{C}_{9} \mathrm{H}_{9} \mathrm{O}_{2}\right]^{-}$.

\subsubsection{Identification of Phenylpropanoids.}

Phenylpropanoids and their derivatives, including monocaffeoylquinic acids, biscaffeoylquinic acids, and caffeoylquinic acid derivatives, were main components widely present in MTBD. Some papers [28] have previously shown that phenylpropanoids have multifaceted effects which include anti-inflammatory, antioxidant, antimicrobial, and antidiabetic activities and exhibit renoprotective, hepatoprotective, and cardioprotective effects. By using the FSCIF strategy, twenty phenylpropanoids were found; the ions at $\mathrm{m} / z$ 191.05 Da and 179.03 Da represented the base peaks of quinic acid, whereas ions at $m / z 161.02 \mathrm{Da}$ and $135.04 \mathrm{Da}$ represented the base peaks of caffeic acid. Chlorogenic acid is an ester of caffeic acid and quinic acid, which indicates that chlorogenic acid contains the feature ions of both caffeic acid and quinic acid. A total of twelve compounds (4, 14, 22, 29, 30, $38,45,55,56,60,61$, and 63 ) were detected by $m / z 191.05 \mathrm{Da}$ and $179.03 \mathrm{Da}$. Compound 22 was tentatively identified as chlorogenic acid, producing $\mathrm{m} / \mathrm{z} 191.0554, \mathrm{~m} / z 179.0341$ (compound 32), $m / z$ 173.0446, $m / z$ 161.0234, $m / z$ 155.0338, $m / z$ 137.0322, $m / z 135.0440$, and $m / z$ 93.0333, which were corresponding to $\left[\mathrm{M}-\mathrm{H}-\mathrm{C}_{9} \mathrm{H}_{6} \mathrm{O}_{3}\right]^{-},\left[\mathrm{M}-\mathrm{H}-\mathrm{C}_{7} \mathrm{H}_{10} \mathrm{O}_{5}\right]^{-},[\mathrm{M}-\mathrm{H}-$ $\left.\mathrm{C}_{9} \mathrm{H}_{6} \mathrm{O}_{3}-\mathrm{H}_{2} \mathrm{O}\right]^{-}, \quad\left[\mathrm{M}-\mathrm{H}-\mathrm{C}_{7} \mathrm{H}_{10} \mathrm{O}_{5}-\mathrm{H}_{2} \mathrm{O}\right]^{-}, \quad\left[\mathrm{M}-\mathrm{H}-\mathrm{C}_{9} \mathrm{H}_{6} \mathrm{O}_{3}-\right.$ $\left.2 \mathrm{H}_{2} \mathrm{O}\right]^{-}, \quad\left[\mathrm{M}-\mathrm{H}-\mathrm{C}_{9} \mathrm{H}_{6} \mathrm{O}_{3}-3 \mathrm{H}_{2} \mathrm{O}\right]^{-}, \quad\left[\mathrm{M}-\mathrm{H}-\mathrm{C}_{7} \mathrm{H}_{10} \mathrm{O}_{5}-\mathrm{CO}_{2}\right]^{-}$, and $\left[\mathrm{M}-\mathrm{H}-\mathrm{C}_{9} \mathrm{H}_{6} \mathrm{O}_{3}-3 \mathrm{H}_{2} \mathrm{O}-\mathrm{CO}_{2}\right]^{-}$. Figure $\mathrm{S} 3-\mathrm{B}$ shows the main cracking pathways of chlorogenic acid. Compounds 16, 32,33 , and 77 were filtered by $m / z 161.02 \mathrm{Da}$ and $135.04 \mathrm{Da}$, which were indicative of caffeic acid derivatives. Take compound $77\left(\mathrm{C}_{11} \mathrm{H}_{12} \mathrm{O}_{4}\right)$ as an example; $[\mathrm{M}-\mathrm{H}]^{-}$at $\mathrm{m} / z$ 207.0662 and the secondary mass spectrometry were detected at $m / z 179.0341$ [M-H-CO] ${ }^{-}, m / z 161.0234$ [M-H-CO- $\left.\mathrm{H}_{2} \mathrm{O}\right]^{-}$, and $m / z 135.0440\left[\mathrm{M}-\mathrm{H}-\mathrm{CO}-\mathrm{CO}_{2}\right]^{-}$, which were tentatively identified as ethyl caffeate. Compounds 32 and 33 also have similar pyrolysis laws.

3.2.4. Identification of Iridoids. The most basic core of iridoids is iridoid alcohol, containing cyclic ethers and alcoholic hydroxyl groups, which imply that the basic skeleton of iridoid glycosides contains a characteristic dihydropyran ring which is cis-connected to a cyclopentane unit structure. A total of 15 iridoids were detected $[\mathrm{M}-\mathrm{H}]^{-}$in negative ion mode. In the $\mathrm{ESI}^{-}$mode, the fragment ion ${ }^{2,7} \mathrm{~F}^{-}$ion at $\mathrm{m} / z$ 101.02 was obtained by the fragmentation of the aglycon part of the excimer ion, which was a characteristic ion to annotate the structure of the excimer ion $[29,30]$. According to the literature [11], the ion at $m / z 147.03$ was the prominent ion of iridoids. Compounds 5, 8, 11, 13, and 40 were detected by characteristic ions 147.03 Da or 101.02 Da. Taking the derivation process of compound 8 as an example, the quasimolecular ion peak of compound 8 was $\mathrm{m} / z 389.1089$ $[\mathrm{M}-\mathrm{H}]^{-}$, yielding a formula of $\mathrm{C}_{16} \mathrm{H}_{22} \mathrm{O}_{11}$. The $[\mathrm{M}-\mathrm{H}]^{-}$ ion of $\mathrm{m} / z 227.0550$ was the absence of glucose neutral fragment from $m / z$ 389.1089. The fragment ions $m / z$ of 209.0356 and 183.0655 were losing one molecule of $\mathrm{H}_{2} \mathrm{O}$ and one molecule of $\mathrm{CO}_{2}$ from $[\mathrm{M}-\mathrm{H}]^{-}$ion of $m / z 227.0550$. Then, the ion of $\mathrm{m} / \mathrm{z} 183.0655$ losses two molecules of $\mathrm{H}_{2} \mathrm{O}$, convert to the fragment ions of $\mathrm{m} / z 165.0543$ and $\mathrm{m} / z$ 147.0285. Consistently, the dehydration of fragment ion $\mathrm{m} / z$ 209.0356 leads to the production of $\mathrm{m} / z$ 191.0553; and the fragment ion $m / z 147.0285$ was decarboxylation of $\mathrm{m} / z$ 191.0553. The cleavage detail of each ion is displayed in Figure S3-C.

In addition, iridoid glycosides are usually connected to a glucose at the $\mathrm{C} 1$ position, so they are easy to lose neutral fragments such as $162.02 \mathrm{Da}$ (glc), $44.01 \mathrm{Da}\left(\mathrm{CO}_{2}\right)$, and 18.01 $\mathrm{Da}\left(\mathrm{H}_{2} \mathrm{O}\right)$ [31]. A total of five compounds $(7,10,21,28$, and 48) were filtered by $162.02 \mathrm{Da}$. Compound 7 $\left(\mathrm{C}_{16} \mathrm{H}_{22} \mathrm{O}_{10}\right)$ showed $[\mathrm{M}-\mathrm{H}]^{-}$at $m / z$ 373.1140; the fragment ions determined from MS/MS spectra were $\mathrm{m} / z 211.0606$

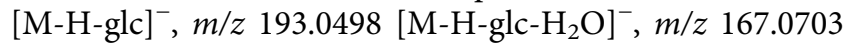
[M-H-glc- $\left.\mathrm{CO}_{2}\right]$, and $m / z 149.0598$ [M-H-glc- $\mathrm{CO}_{2}-\mathrm{H}_{2} \mathrm{O}$ ]; $\mathrm{m} / z 211.0940(373.1140 \mathrm{Da}-162.02 \mathrm{Da})$ were characteristic fragments of compound 7, which was tentatively annotated as geniposidic acid. To sum up, the iridoids were easier to lose the glucose neutral fragment ion 162.02 Da and obtain aglycon fragment ions and then the aglycon ions decarboxylated or dehydrated to become a series of fragments.

3.2.5. Identification of Lignans. A large number of the bisepoxylignans and monoepoxylignans combine with glucose to form monoglycoside or diglycoside. Therefore, the majority of them could lose glycosyl and methyl neutral 
fragments first and then lose one or two molecular of $\mathrm{CH}_{2} \mathrm{O}$ and finally formed $151.03 \mathrm{Da}$. Therefore, characteristic ion fragment $151.03 \mathrm{Da}$ was used to annotate lignans. Compounds $31,44,52,53,101$, and 132 were detected by FSCIF with $151.03 \mathrm{Da}$. Compound 44 showed $[\mathrm{M}-\mathrm{H}]^{-}$at $\mathrm{m} / z$ 681.2400; the fragment ions $\mathrm{m} / z 519.5070, \mathrm{~m} / z 357.1346$, and $\mathrm{m} / z 151.0390$ were corresponding to [M-H-glc] ${ }^{-},[\mathrm{M}-\mathrm{H}-\mathrm{glc}-$ glc $]^{-}$, and [M-H-glc-glc- $\left.\mathrm{C}_{12} \mathrm{H}_{14} \mathrm{O}_{3}\right]^{-}$. Subsequently, compounds 37,39, 138, and 139 were filtered by NLF with ions of 162.02 Da, 44.01 Da, or 18.01 Da.

3.2.6. Other Compounds. A total of 11 polyphenols $(3,6,9$, $17,18,20,23,24,25,34$, and 35) were recognized by FSNLF analysis. Because of the presence of hydroxyl and carboxyl groups, these compounds were filtered by $18.01 \mathrm{Da}\left(\mathrm{H}_{2} \mathrm{O}\right)$ and 44.01 Da $\left(\mathrm{CO}_{2}\right)$. In addition, six other compounds were identified by comparison with the literature.

\subsection{Quantification of 19 Major Compounds in MTBD.} The 19 compounds quantified were the screening of osteoporosis targets by network pharmacology in the early stage of our laboratory, and then the representative and top ranked compounds were selected. Methodology analysis showed that the assay method of 19 compounds (including three pairs of isomers) had good repeatability and stability.

3.3.1. Specificity. The extracted ion chromatograms (EICs) of blank sample, standard mixture sample, and MTBD extracts sample are presented in Figure 4. Nineteen compounds in MTBD extracts were separated within 25 minutes, where baseline separation of each compound was achieved and no obvious signal noises occurred around determinate peak. Additionally, no interferences were detected between the three isomers.

3.3.2. Linearity and Lower Limit of Quantification. Three batches of standard curve solutions with six different concentrations were prepared. The typical standard curves were assessed by using DAS 2.0 software with the quadratic weight $\left(W=1 / C^{2}\right)$. The dependent variable was the ratio of the peak area of each analyte to the peak area of the internal standard, while the independent variable was set as the concentration value of each analyte; the least square regression was used to construct the standard curve equation. The standard curves and correlation coefficients are listed in Table 2, proving the calibration curves of the components with a good linearity over the studied concentration range.

The lower limit of quantification (LLOQ) for each analyte was all with signal-to-noise ratio higher than 10 , which was sufficient to perform quantitative studies of MTBD extracts.

3.3.3. Precision and Accuracy. Three batches of quality control samples were prepared according to three concentration levels. Each concentration was analyzed with $6 \mathrm{du}-$ plications. The intraday precision values were between $1.13 \%$ and $6.66 \%$, and the interday ones were between $2.42 \%$ and
$10.62 \%$ and accuracy ranged from $86.11 \%$ to $114.27 \%$. The above results demonstrated the acceptable precision and accuracy of the present method.

3.3.4. Repeatability and Stability. Six MTBD sample extracts were prepared on the same day according to Section 2.2. The repeatability of 19 components was within $6.26 \%$ relative standard deviation ( $R S D)$.

Sample stability was investigated after the extracts were kept at room temperature for $0 \mathrm{~h}, 6 \mathrm{~h}, 12 \mathrm{~h}$, and $24 \mathrm{~h}$. The stability results of 19 compounds are summarized in Table 3; the acceptability of the data was within $3.92 \%$ deviation from the $0 \mathrm{~h}$ sample values, which indicated that a large number of samples could be stable in each analytical run.

3.4. Application to Samples Modified Tabusen-2 Decoction (MTBD). The method established above was successfully utilized for quantitative studies of MTBD extracts, as shown in Table S2. Eight batches of MTBD samples prepared with different herb sources were determined by using the above mature method. The herb formulation of each batch is listed in Table S3. There is an indication of the fact that the concentrations of 19 compounds varied significantly in MTBD extracts; the content of flavonoids was the highest, followed by saponins (Figure S4), which attracted the attention of herb quality in picking as well as in circulating during the market. It can be seen from the quantitative research results of different batches of MTBD that we need to strictly control the quality of herb because this is the guarantee of their clinical efficacy and safety.

\section{Discussion}

Although the isolation and purification before biological activity evaluation are a traditional strategy of exploring material basis in TMM, the time-consuming and labor-intensive characteristics cannot be neglected. In quantitative experiments, Ultraviolet (UV) detector is exceedingly common for flavonoids, phenylpropanoids, and other UV-absorbing compounds [32], while it is not applicable to the analysis of saponin. Although the detection of saponin could be enabled by evaporative light scattering detector (ELSD), the sensitivity during the test procedure should also be taken into account [33]. Herein, in order to shorten the analysis time, improve the analysis sensitivity, and simultaneously determine UV-absorbing compounds and non-UV-absorbing compounds, high performance liquid chromatography coupled with mass spectrometry (HPLC-Q-Exactive MS/MS spectrometer) approach [34], as a high efficiency, is employed in this study to separate and identify the material basis in MTBD. Additionally, the existence of isomer (ICGAA with 1,5-DQA, GE with APG, LT with KPF) in MTBD increases the difficulty of separation and analysis [35]. The chromatographic conditions in quantitative analysis need to be optimized carefully during the present research.

In order to achieve better separation effect for three pairs of isomers in MTBD, the mobile phase was screened in this experiment. The peak of each component was with 


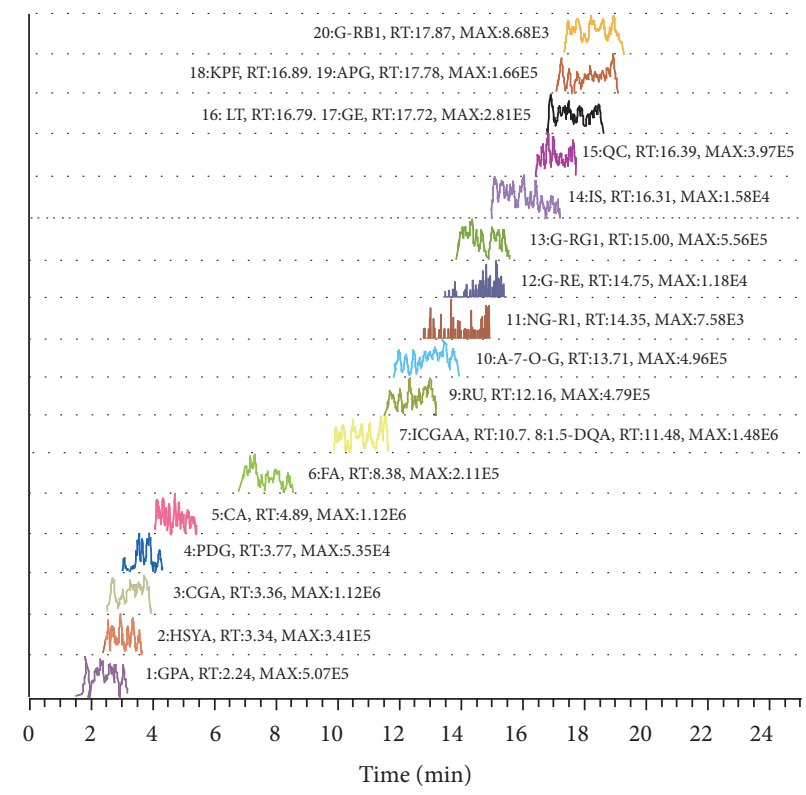

(a)

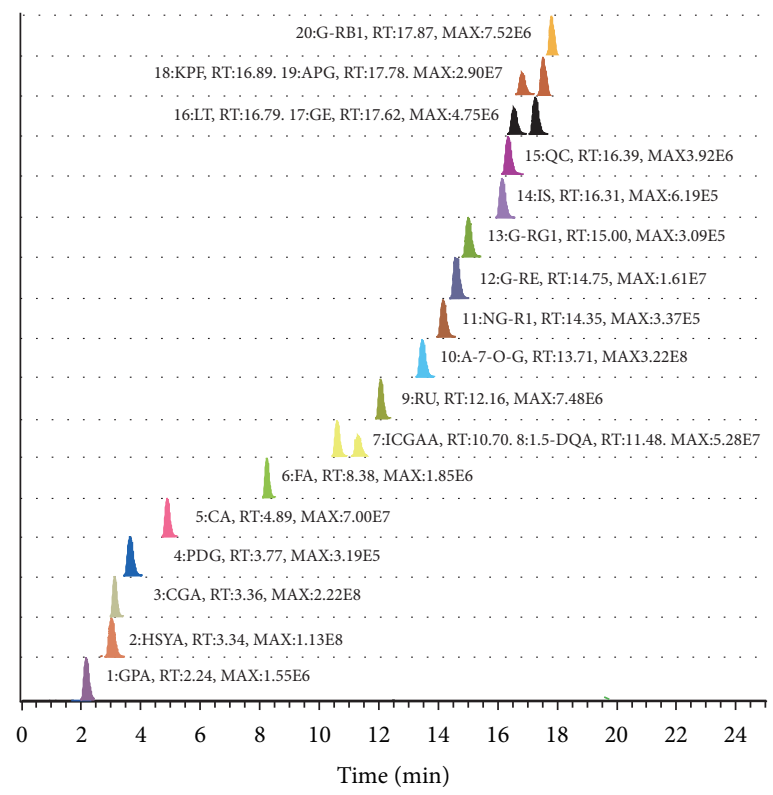

(b)

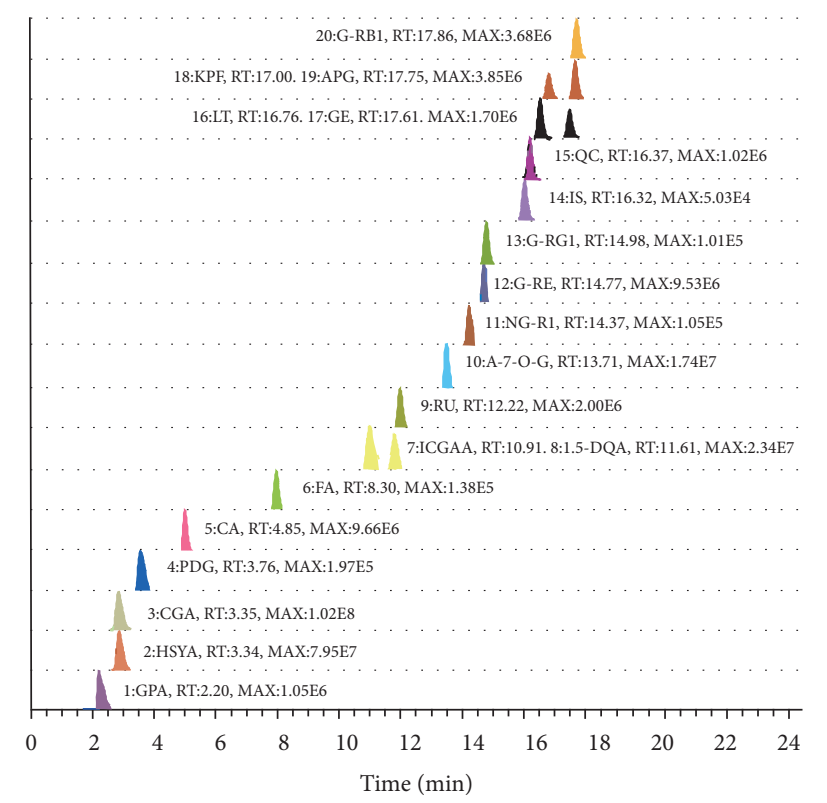

(c)

FIGURE 4: Representative chromatograms of (a) blank, (b) 19 standard samples, and (c) 19 compounds in MTBD.

symmetrical shape and no tailing phenomenon. Additionally, the influence of column temperature and flow rate was considered, and a better separation was achieved under column temperature of $30^{\circ} \mathrm{C}$ and flow rate of $0.3 \mathrm{~mL} / \mathrm{min}$. A variety of chromatographic columns were also optimized in this study. Compared with ACE C18-PFP column $(100 \times 3.0 \mathrm{~mm}$ ID, $3 \mu \mathrm{m})$, Grace Alltima C18 column $(250 \mathrm{~mm} \times 4.6 \mathrm{~nm}, 5 \mu \mathrm{m})$, HITACHI LaChrom C18 column $(250 \mathrm{~mm} \times 4.6 \mathrm{~mm}$ ID, $5 \mu \mathrm{m})$, and Thermo ODS-2 HYPERSIL column $(250 \mathrm{~mm} \times 4.6 \mathrm{~mm}, 5 \mu \mathrm{m})$, ACE C18PFP column had better separation and resolution, especially for the three isomers.
It was found through analysis that the contents of the 19 components differ in MTBD prepared from different batches of crude drugs; this might be because the crude drugs of different batches were different in origin, growing environments, and harvest time. This has aroused our attention in all aspects of picking and transportation. The presence of moisture will affect the determination of the content of the active ingredients in the medicinal materials. Therefore, the near-infrared method was used in the study to detect the moisture content in the relevant medicinal materials to ensure the final quantitative accuracy of the effective ingredients [36]. Refluxing was used 
TABLE 2: Calibration curves, linear range, $r^{2}$, and LOQs of 19 compounds in MTBD.

\begin{tabular}{|c|c|c|c|c|}
\hline Compound & Calibration curves & Linear range $(\mu \mathrm{g} / \mathrm{mL})$ & $r^{2}$ & LLOQ $(\mu \mathrm{g} / \mathrm{mL})$ \\
\hline ICGAA & $y=647.937 x+1.476$ & $4.800-192.000$ & 0.9994 & 4.800 \\
\hline 1,5-DQA & $y=364.018 x-0.810$ & $6.100-244.000$ & 0.9991 & 6.810 \\
\hline GE & $y=22386.297 x-0.191$ & $0.023-0.920$ & 0.9982 & 0.025 \\
\hline APG & $y=29960.140 x-0.562$ & $0.065-2.600$ & 0.9989 & 0.073 \\
\hline $\mathrm{LT}$ & $y=15454.517 x-0.090$ & $0.015-0.600$ & 0.9986 & 0.015 \\
\hline KPF & $y=9208.139 x-0.051$ & $0.010-0.400$ & 0.9983 & 0.010 \\
\hline QC & $y=8437.051 x-0.062$ & $0.011-0.440$ & 0.9989 & 0.011 \\
\hline A-7-O-G & $y=6901.196 x+11.968$ & $4.200-168.000$ & 0.9962 & 4.200 \\
\hline RU & $y=2560.803 x-0.093$ & $0.230-9.200$ & 0.9992 & 0.250 \\
\hline HSYA & $y=1104.734 x-0.935$ & $9.830-392.000$ & 0.9984 & 10.930 \\
\hline NG- $R_{1}$ & $y=8.300 x-0.016$ & $2.400-96.000$ & 0.9964 & 2.400 \\
\hline G-Re & $y=730.821 x+0.137$ & $1.010-40.400$ & 0.9963 & 1.010 \\
\hline $\mathrm{G}-\mathrm{Rg}_{1}$ & $y=21.700 x+0.029$ & $6.500-260.000$ & 0.9983 & 6.700 \\
\hline $\mathrm{G}-\mathrm{Rb}_{1}$ & $y=93.320 x+0.128$ & $5.660-226.400$ & 0.9979 & 5.830 \\
\hline $\mathrm{CA}$ & $y=183840.263 x-8.999$ & $0.260-10.400$ & 0.9985 & 0.260 \\
\hline FA & $y=2322.874 x-0.525$ & $0.390-15.600$ & 0.9992 & 0.410 \\
\hline GPA & $y=119.544 x-0.011$ & $0.800-32.000$ & 0.9987 & 0.800 \\
\hline CGA & $y=3414.355 x-5.245$ & $5.500-220.000$ & 0.9978 & 5.660 \\
\hline PDG & $y=10.556 x-0.018$ & $2.180-87.200$ & 0.9987 & 2.180 \\
\hline
\end{tabular}

TABle 3: Precision, repeatability, stability, and accuracy of 19 compounds in MTBD.

\begin{tabular}{|c|c|c|c|c|c|c|c|c|c|c|}
\hline \multirow[t]{2}{*}{ Compound } & \multicolumn{3}{|c|}{$\begin{array}{l}\text { Interday precision } \\
\quad(\mathrm{RSD}, n=3)\end{array}$} & \multicolumn{3}{|c|}{$\begin{array}{l}\text { Intraday precision } \\
\quad(\mathrm{RSD}, n=3)\end{array}$} & \multirow{2}{*}{$\begin{array}{c}\text { Repeatability } \\
(\mathrm{RSD}, n=6, \%)\end{array}$} & \multirow{2}{*}{$\begin{array}{c}\text { Stability } \\
(\mathrm{RSD}, n=4, \%)\end{array}$} & \multicolumn{2}{|c|}{$\begin{array}{l}\text { Accuracy } \\
(n=6, \%)\end{array}$} \\
\hline & Low & Middle & High & Low & Middle & High & & & Recovery & RSD \\
\hline ICGAA & 4.74 & 5.61 & 6.45 & 1.24 & 2.34 & 3.39 & 2.62 & 3.13 & 107.36 & 1.76 \\
\hline 1,5-DQA & 3.37 & 4.31 & 5.18 & 1.13 & 0.59 & 1.63 & 6.26 & 3.48 & 106.46 & 2.11 \\
\hline GE & 2.42 & 7.24 & 4.87 & 2.29 & 2.79 & 0.90 & 2.30 & 2.68 & 106.41 & 2.06 \\
\hline APG & 3.34 & 5.34 & 3.64 & 3.22 & 2.58 & 2.82 & 1.70 & 2.18 & 106.73 & 2.23 \\
\hline LT & 4.05 & 7.84 & 4.53 & 3.13 & 2.81 & 1.09 & 4.75 & 3.92 & 98.21 & 3.01 \\
\hline $\mathrm{KPF}$ & 9.47 & 9.00 & 7.98 & 2.18 & 1.54 & 5.51 & 4.94 & 3.60 & 103.12 & 2.37 \\
\hline QC & 8.67 & 6.94 & 5.52 & 6.66 & 2.25 & 1.35 & 4.68 & 2.81 & 103.42 & 4.10 \\
\hline A-7-O-G & 4.17 & 3.42 & 7.38 & 4.15 & 3.05 & 3.13 & 2.09 & 2.38 & 104.56 & 1.87 \\
\hline RU & 4.03 & 3.23 & 3.42 & 1.86 & 1.92 & 2.57 & 2.99 & 2.34 & 105.39 & 0.92 \\
\hline HSYA & 3.81 & 4.97 & 5.67 & 2.25 & 1.13 & 4.78 & 3.40 & 2.56 & 104.80 & 1.13 \\
\hline NG-R1 & 8.22 & 4.19 & 5.85 & 1.63 & 2.07 & 4.55 & 4.05 & 0.75 & 104.87 & 2.81 \\
\hline G-Re & 6.50 & 3.92 & 5.41 & 3.17 & 1.38 & 2.47 & 4.02 & 3.23 & 107.35 & 1.73 \\
\hline $\mathrm{G}-\mathrm{Rg}_{1}$ & 6.10 & 7.59 & 6.12 & 5.08 & 4.64 & 4.80 & 3.27 & 2.26 & 104.64 & 1.59 \\
\hline $\mathrm{G}-\mathrm{Rb}_{1}$ & 10.62 & 3.37 & 3.61 & 2.94 & 2.71 & 3.47 & 4.50 & 2.99 & 106.94 & 2.54 \\
\hline $\mathrm{CA}$ & 6.29 & 5.83 & 4.08 & 5.45 & 3.62 & 4.31 & 2.15 & 2.43 & 101.10 & 0.53 \\
\hline FA & 8.76 & 7.21 & 7.61 & 3.90 & 4.15 & 2.40 & 2.94 & 3.74 & 105.71 & 2.10 \\
\hline GPA & 8.36 & 6.89 & 10.52 & 2.64 & 0.54 & 4.11 & 3.82 & 3.59 & 107.60 & 3.01 \\
\hline CGA & 2.74 & 5.12 & 2.82 & 2.52 & 1.25 & 3.50 & 2.99 & 2.24 & 106.59 & 2.11 \\
\hline PDG & 6.95 & 7.32 & 7.71 & 2.69 & 2.78 & 4.11 & 2.67 & 1.97 & 92.08 & 4.57 \\
\hline
\end{tabular}

to prepare the MTBD in the present study [37]. Furthermore, some literatures [38-42] have carried out assays on HSYA, RU, QC, G-Rb, G-RG , NG-R $_{1}, \mathrm{G}-\mathrm{Re}, \mathrm{FA}, \mathrm{LT}$, $\mathrm{KPF}, \mathrm{APG}$, and GE; but the HPLC-Q-Exactive MS/MS spectrometer approach displayed distinct superiority with desirable resolution and Lower LLOQ. The previous literature $[43,44]$ measured the content of CA, 1.5-DQA, GPA, PDG, and CGA, but it took too long (60 minutes) and restricted its modern development. Some studies $[45,46]$ have shown the contents of ICGGA and A-7-O-G; on this basis, we can have a wider linear range and have greater reference value for the formulation of the content of different batches of samples.

\section{Conclusions}

Based on HPLC-Q-Exactive MS/MS spectrometer with FSCIF approach to rapid detection of structure fragment and quantification of major representative components in MTBD, 143 compounds with seven chemical categories were unambiguously or tentatively identified. This study not only enriched the cleavage law of MTBD compounds but also established an approach for the accurate search and discovery of active components from complex mixtures. The repeatability, accuracy, stability, linearity, recoveries, and reproducibility of quantitative analysis all meet the criteria for acceptability of quantitative studies. 
The determination of 19 compounds in MTBD extracts in different batches was obtained to monitor the quality of each prescription, which facilitates the better development of quality evaluation technique in MTBD and will help for further exploration of quality control of MTBD. The 19 compounds determined based on the qualitative and quantitative results are the major components of the MTDB. This experiment can provide a research foundation for subsequent pharmacokinetic studies and formulation of quality standards.

All in all, we compared the differences in the content of the same compound in the same herbs. Our quantitative method can determine 19 compounds in a short time (25 minutes), with a wider linear range and lower LLOQ. On the other hand, we compared the content difference of the same compound in different herbs, and the content fluctuation range is relatively large, which may be related to the processing, compatibility, and the changes in the decocting process of herbs. The content range of the 19 compounds that we have measured can provide the fluctuation range of the compound content when formulating quality standards in the future and help formulate content determination standards for preparations. This qualitative and quantitative analysis of MTBD could provide a new tool for the quality control of this preparation or its related TCM.

\section{Data Availability}

The methodological data and structural data used to support the findings of this study are included within the article. The cleavage pathways data used to support the findings of this study are included within the Supplementary Materials.

\section{Disclosure}

This article has become a preprint: https://doi.org/10.21203/ rs.3.rs-829266/v1.

\section{Conflicts of Interest}

The authors declare that there are no conflicts of interest regarding the publication of this paper.

\section{Authors' Contributions}

Yu Zhao and Xin Dong contributed equally to this work. All authors read and approved the final manuscript.

\section{Acknowledgments}

This work was financially supported by the National Natural Science Foundation of China (81860756 and 81960758), Student Innovation and Entrepreneurship Fund Project of China (202010132003 and 202110132029), Innovation Guide Project (02039001), and Inner Mongolia Autonomous Region Higher Education Science Research Project (NJZY19099).

\section{Supplementary Materials}

Table S1: the final concentration of 19 standard solutions. Table S2: the content of 19 compounds in the MTBD medicinal measure $(\mu \mathrm{g}, X \pm \mathrm{SD}, n=3)$. Table S3: different sources of medicinal materials. Table S4: quality control concentration levels of 19 compounds. Figure S1: structures of 143 compounds. (A) Structure of flavonoids. (B) Structure of phenylpropanoids. (C) Structure of lignans. (D) Structure of iridoids. (E) Structure of polyphenol. (F) Structure of saponins. (G) Structure of other type compounds. Figure S2: the cracking pathways of (A) ginsenoside $R b_{1}$, (B) notoginsenoside $R_{1}$, and (C) 3-( $\beta$-D-glucopyranosyl- $\beta$-Dglucopyranosyl)-20-O-(6-O-malonyl- $\beta$-D-glucopyranosyl- $\beta$ D-glucopyranosyl)-3 $\beta, 12 \beta, 20(\mathrm{~S})$-trihydroxydammar-24-ene. Figure S3: the cracking pathways of (A) rutin, (B) chlorogenic acids, (C) deacetyl asperulosidic acid. Figure S4: the content of eight batches of medicinal materials. (Supplementary Materials)

\section{References}

[1] J. Q. Wang, X. Dong, F. X. Ma et al., "Metabolomics profiling reveals Echinops latifolius Tausch improves the trabecular micro-architecture of ovariectomized rats mainly via intervening amino acids and glycerophospholipids metabolism," Journal of Ethnopharmacology, vol. 260, Article ID 113018, 2020.

[2] J. H. Xie, Z. Y. Tan, Y. M. Guo, H. Yang, and G. Y. Hu, "Quality standard control of Modified Tabusen-2 decoction and TLC identification method of Eucommia-containing preparations," Chinese Patent Medicines, vol. 3, pp. 632-636, 2017.

[3] F. Yang, X. Dong, F. Ma et al., "The interventional effects of Tubson-2 Decoction on ovariectomized rats as determined by a combination of network pharmacology and metabolomics," Frontiers in Pharmacology, vol. 11, Article ID 581991, 2020.

[4] J. Y. Li and H. C. Song, "Orthogonal design to optimize the extraction and molding process of Yushang Jiegu capsules," Journal of Medicine \& Pharmacy of Chinese Minorities, vol. 5, no. 5, pp. 53-55, 2010.

[5] L. Gao and Q. Shi, "Determination of ursolic acid in yushang jiegu capsules by HPLC," Chinese Traditional Patent Medicine, vol. 29, no. 29, pp. 398-399, 2006.

[6] J. Zhao, C. Y. Dong, J. P. Shi et al., "Micro-histomorphometry study of Mongolian medicine echinops sphaerocephalus L. on postmenopausal osteoporosis," Chinese Journal of Osteoporosis, vol. 26, no. 7, pp. 972-977, 2020.

[7] M. Liu, G. Ye, Y. J. Cui, A. Y. Zhang, Y. Y. Zhao, and D. A. Guo, "Study on the chemical constituents of the aboveground parts of Huadong Echinops latifolius Tausch," Chinese Traditional and Herbal Drugs, vol. 33, no. 1, pp. 18-20, 2002.

[8] J. Q. Wang, Study on the Mechanism of Mongolian Medicine Nitraria Glabra against Osteoporosis Based on Network Pharmacology, Inner Mongolia Medical University, Hohhot, China, 2019.

[9] X. Gong, Q. Luan, X. Zhou, Y. Zhao, and C. Zhao, "UHPLCESI-MS/MS determination and pharmacokinetics of pinoresinol glucoside and chlorogenic acid in rat plasma after oral administration of Eucommia ulmoides Oliv extract," Biomedical Chromatography, vol. 31, no. 11, 2017. 
[10] C. Y. Wang, L. Tang, J. W. He, J. Li, and Y. Z. Wang, "Ethnobotany, phytochemistry and pharmacological properties of eucommia ulmoides: a review," The American Journal of Chinese Medicine, vol. 47, no. 2, pp. 259-300, 2019.

[11] M. He, J. Jia, J. Li et al., "Application of characteristic ion filtering with ultra-high performance liquid chromatography quadrupole time of flight tandem mass spectrometry for rapid detection and identification of chemical profiling in Eucommia ulmoides Oliv," Journal of Chromatography A, vol. 1554, no. 54, pp. 81-91, 2018.

[12] L. L. Zhang, K. Tian, Z. H. Tang et al., "Phytochemistry and pharmacology of carthamus tinctorius L," The American Journal of Chinese Medicine, vol. 44, no. 2, pp. 197-226, 2016.

[13] X. Duan, L. Pan, D. Peng et al., "The analysis of the active components and metabolites of taohong siwu decoction based on ultra performance liquid chromatography quadrupole time-of-flight mass spectrometry," Journal of Separation Science, vol. 43, no. 22, pp. 4131-4147, 2020.

[14] Z. Ju, J. Li, Q. Lu, Y. Yang, L. Yang, and Z. Wang, "Identification and quantitative investigation of the effects of intestinal microflora on the metabolism and pharmacokinetics of notoginsenoside Fc assayed by liquid chromatography/ electrospray ionization tandem mass spectrometry," Journal of Separation Science, vol. 42, no. 9, pp. 1740-1749, 2019.

[15] Y. Ma, B. X. Huang, W. W. Tang, P. Li, and J. Chen, "Characterization of chemical constituents and metabolites in rat plasma after oral administration of San Miao Wan by ultra-high performance liquid chromatography tandem Q-Exactive Orbitrap mass spectrometry," Journal of Chromatography B, vol. 2021, Article ID 122793, 2021.

[16] J. Zhang, Z. H. Huang, X. H. Qiu, Y. M. Yang, D. Y. Zhu, and W. Xu, "Neutral fragment filtering for rapid identification of new diester-diterpenoid alkaloids in roots of Aconitum carmichaeli by ultra-high-pressure liquid chromatography coupled with linear ion trap-orbitrap mass spectrometry," PLoS One, vol. 7, no. 12, p. e52352, 2012.

[17] X. Qiao, X.-H. Lin, S. Ji et al., "Global profiling and novel structure discovery using multiple neutral loss/precursor ion scanning combined with substructure recognition and statistical analysis (MNPSS): characterization of terpene-conjugated curcuminoids in curcuma longa as a case study," Analytical Chemistry, vol. 88, no. 1, pp. 703-710, 2016.

[18] B. J. Waldner, R. Machalett, S. Schönbichler, M. Dittmer, M. M. Rubner, and D. Intelmann, "Fast evaluation of herbal substance class composition by relative mass defect plots," Analytical Chemistry, vol. 92, no. 19, pp. 12909-12916, 2020.

[19] L. L. Fu, H. Ding, and L. F. Han, "Simultaneously targeted and untargeted multicomponent characterization of erzhi pill by offline two-dimensional liquid chromatography/quadrupoleorbitrap mass spectrometry," Journal of Chromatography A, vol. 1584, no. 84, pp. 87-96, 2019.

[20] L. L. He, H. Jiang, T. H. Lan et al., "Chemical profile and potential mechanisms of huo-tan-chu-shi decoction in the treatment of coronary heart disease by UHPLC-Q/TOF-MS in combination with network pharmacology analysis and experimental verification," The Journal of Chromatography $B$ Analytical Technologies in the Biomedical and Life Sciences, vol. 15, no. 1175, Article ID 122729, 2021.

[21] X. G. Liu, J. S. Li, S. X. Feng et al., "A high-resolution MS/MS based strategy to improve xenobiotic metabolites analysis by metabolic pathway extension searching combined with parallel reaction monitoring: flavonoid metabolism in wound site as a case," The Journal of Chromatography B Analytical
Technologies in the Biomedical and Life Sciences, vol. 1162, Article ID 122470, 2021.

[22] J. H. Xie, Z. Y. Tan, Y. M. Guo, H. Yang, and G. Y. Hu, "Quality standard control of lanhong capsules and TLC identification method of eucommia-containing preparations," Chinese Traditional Patent Medicine, vol. 03, no. 39, pp. 194-198, 2017.

[23] X. W. Hua, Lanhong Capsule Quality Standard and Study on the Fingerprint of Lancitou, Xinjiang Medical University, Ürümqi, China, 2014.

[24] X. W. Hua, Y. Li, X. M. Cheng, H. Yang, G. Y. Hu, and C. H. Wang, "Quality control method of Panax notoginseng in lanhong capsules," Chinese Traditional Patent Medicine, vol. 36, no. 7, pp. 1497-1501, 2014.

[25] Y. Ling and Q. Zhang, "Structural characterisation and screening of triterpene saponins in the bark of Ilex rotunda using high-performance liquid chromatography coupled to electrospray ionisation and quadrupole time-of-flight mass spectrometry," Phytochemical Analysis, vol. 32, no. 3, pp. 395-403, 2021.

[26] M. Yoshikawa, T. Morikawa, K. Yashiro, T. Murakami, and H. Matsuda, "Bioactive saponins and glycosides. XIX. Notoginseng(3):immunological adjuvant activity of notoginsenosides and related saponins: structures of notoginsenosides- $\mathrm{L},-\mathrm{M}$, and -N from the roots of Panax notoginseng (Burk.) F. H. Chen," Chemical and Pharmaceutical Bulletin, vol. 49, no. 11, pp. 1452-1456, 2001.

[27] W. Z. Yang, X. J. Shi, C. L. Yao et al., "A novel neutral loss/ product ion scan-incorporated integral approach for the untargeted characterization and comparison of the carboxylfree ginsenosides from panax ginseng, panax quinquefolius, and panax notoginseng," Journal of Pharmaceutical and Biomedical Analysis, vol. 2020, Article ID 112813, 2020.

[28] B. Vanholme, I. El Houari, and W. Boerjan, "Bioactivity: phenylpropanoids' best kept secret," Current Opinion in Biotechnology, vol. 56, pp. 156-162, 2019.

[29] X. Wang, W. Wu, J. Zhang et al., "An integrated strategy for holistic quality identification of Chinese patent medicine: liuwei Dihuang Pills as a case study," Phytochemical Analysis, vol. 32, no. 2, pp. 183-197, 2021.

[30] X. Wang, C. Wu, M. Xu, C. Cheng, Y. Liu, and X. Di, "Optimisation for simultaneous determination of iridoid glycosides and oligosaccharides in Radix Rehmannia by microwave assisted extraction and HILIC-UHPLC-TQ-MS/ MS," Phytochemical Analysis, vol. 31, no. 3, pp. 340-348, 2020.

[31] F. Shi, C. Tong, C. He, S. Shi, Y. Cao, and Q. Wei, "Diagnostic ion filtering targeted screening and isolation of anti-inflammatory iridoid glycosides from Hedyotis diffusa," Journal of Separation Science, vol. 44, no. 13, pp. 2612-2619, 2021.

[32] T. H. Sani, M. Hadjmohammadi, and M. H. Fatemi, "Extraction and determination of flavonoids in fruit juices and vegetables using $\mathrm{Fe}_{3} \mathrm{O}_{4} / \mathrm{SiO}_{2}$ magnetic nanoparticles modified with mixed hemi/admicelle cetyltrimethylammonium bromide and high performance liquid chromatography," Journal of Separation Science, vol. 43, no. 7, pp. 1224-1231, 2020.

[33] P. Yang, M. Zhou, C. Zhou, Q. Wang, F. Zhang, and J. Chen, "Separation and purification of both tea seed polysaccharide and saponin from camellia cake extract using macroporous resin," Journal of Separation Science, vol. 38, no. 4, pp. 656-662, 2015.

[34] M. Teich, M. Schmidtpott, D. van Pinxteren, J. Chen, and H. Herrmann, "Separation and quantification of imidazoles in atmospheric particles using LC-Orbitrap-MS," Journal of Separation Science, vol. 43, no. 8, pp. 577-589, 2020. 
[35] N. Li, X. Dong, F. Ma et al., "Pharmacokinetics study of 16 active ingredients from Tabson-2 decoction in normal and d-galactose induced osteoporosis rats by liquid chromatography-tandem mass spectrometry," Journal of Separation Science, vol. 43, no. 18, pp. 3702-3713, 2020.

[36] Y. F. Zhou, Z. X. Yang, and L. Y. Dong, "Rapid determination of moisture and ethanol extract content in Panax notoginseng by NIRS," Drug Evaluation Research, vol. 41, no. 11, pp. 1994-1999, 2018.

[37] M. C. Wei, Y. C. Yang, H. F. Chiu, and H. Hong, "Development of a hyphenated procedure of heat-reflux and ultrasound-assisted extraction followed by RP-HPLC separation for the determination of three flavonoids content in Scutellaria barbata D. Don," Journal of Chromatography B: Analytical Technologies in the Biomedical and Life Sciences, vol. 940, pp. 126-134, 2013.

[38] J. Wu, H. J. Yang, and P. Zhou, "Determination of 5 components in Carthamus tinctorius L," Asia-Pacific Traditional Medicine, vol. 15, no. 11, 2019.

[39] C. L. Yao, W. Z. Yang, and W. Y. Wu, "Simultaneous quantitation of five Panax notoginseng saponins by multi heart-cutting two-dimensional liquid chromatography: method development and application to the quality control of eight Notoginseng containing Chinese patent medicines," Journal of Chromatography A, vol. 1402, pp. 71-81, 2015.

[40] Y. Z. Wang, "Simultaneous determination of paeoniflorin, hydroxysafflor yellow A and ferulic acid in Xielong Liquor by HPLC," Chinese Traditional Patent Medicine, vol. 34, no. 10, pp. 1925-1928, 2012.

[41] J. J. Wang, X. M. Zhang, and H. L. Wang, "Simultaneous determination of quercetin, luteolin, kaempferol and apigenin in plantago asiatica L. by reversed-phase high performance liquid chromatography," Chinese Traditional Patent Medicine, vol. 31, no. 5, pp. 772-775, 2009.

[42] J. B. Wang, P. Guo, X. B. Zhao, and Y. H. Xie, "Determination of genistein in fructus sophorae extract by RP-HPLC," Chinese Traditional and Herbal Drugs, vol. 35, no. 4, pp. 402-403, 2004.

[43] J. Sun, Y. Lu, W. Y. Xiang, X. Cao, Z. P. Gong, and A. M. Wang, "Simultaneously determines the content of 6 active ingredients in Eucommia ulmoides Olivers by UPLC," Natural Product Research and Development, vol. 28, pp. 874-879, 2016.

[44] J. P. Gao, L. Yang, N. Yang et al., "Study on quality of Mongolia medicinal materials flos echinopsis based on HPLC characteristic chromatogram and determination of four caffeic acid derivatives," Journal of Chinese Medicinal Materials, vol. 41, no. 7, pp. 1641-1645, 2018.

[45] M. Hua, W. Bi, J. Chen et al., "The determination of isochlorogenic acid A, B, and C in Lonicera japonica by HPLC," Journal of West China Forestry Science, vol. 46, no. 4, pp. 73-78, 2017.

[46] Y. N. He, Y. G. Zhao, D. L. Yang, D. G. Zhang, and C. M. Wang, "Determination and comparison of luteoloside, apigenin-7-O- $\beta$-D-glucosidase, and luteolin in Humulus scandens from different habitats in Hebei province," Chinese Traditional and Herbal Drugs, vol. 47, no. 20, pp. 3707-3711, 2016. 